\title{
A Fault-Tolerant Location Approach for Transient Voltage Disturbance Source Based on Information Fusion
}

\author{
Guoqing Weng ${ }^{1}$, Feiteng Huang ${ }^{1}$, Jun Yan ${ }^{2}$, Xiaodong Yang ${ }^{1}$, Youbing Zhang ${ }^{1}$ and Haibo He ${ }^{2, *}$ \\ 1 College of Information Engineering, Zhejiang University of Technology, Hangzhou 310023, China; \\ wgq@zjut.edu.cn (G.W.); hfate@zjut.edu.cn (F.H.); yang_xd90@163.com (X.Y.); \\ youbingzhang@zjut.edu.cn (Y.Z.) \\ 2 Department of Electrical, Computer, and Biomedical Engineering, University of Rhode Island, \\ Kingston, RI 02881, USA; jyan@ele.uri.edu \\ * Correspondence: he@ele.uri.edu; Tel.: +1-401-874-5844 \\ Academic Editor: Silvio Simani \\ Received: 15 July 2016; Accepted: 14 December 2016; Published: 20 December 2016
}

\begin{abstract}
This paper proposed a fault-tolerant approach based on information fusion (IF) to automatically locate the transient voltage disturbance source (TVDS) in smart distribution grids. We first defined three credibility factors that will influence the reliability of the direction-judgments at each power quality monitor (PQM). Then we proposed two rules of influence and a verification factor for the distributed generation (DG) integration. Based on the two sets of direction-judgment criteria, a novel decision-making method with fault tolerance based on the IF theory is proposed for automatic location of the TVDS. Three critical schemes, including credibility fusion, conflict weakening, and correction for DG integration, have been integrated in the proposed fusion method, followed by a reliability evaluation of the location results. The proposed approach was validated on the IEEE 13-node test feeder, and the TVDS location results demonstrated the effectiveness and fault tolerance of the IF based approach.
\end{abstract}

Keywords: transient voltage disturbance source; automatic location; direction-judgment; fault tolerance; DG integration; information fusion

\section{Introduction}

With the increasing amount of sensitive loads and the rapid development of electrical power market in smart distribution grids, the intelligent diagnosis of the power quality (PQ) has drawn growing attention [1]. While the classification of PQ disturbance types has been the focus in many fruitful studies [2-4], the accurate location of a power quality disturbance source remains a problem less studied. Nevertheless, the location plays a critical role in determining the cause, responsibility, and effective mitigation strategies [5]. According to statistics, transient voltage disturbance events, mainly the voltage sag and voltage swell, account for the highest portion of disturbances compared to other PQ events [6]. There is an urgent need for advanced approaches for automatic location of transient voltage disturbance source (TVDS) in smart distribution grids.

With the recent progress in networked power quality monitoring system (NPQMS) [7], location strategies of TVDS based on graph theory and matrix algorithm have been developed [5,8,9]. Compared with other strategies based on pattern recognition and artificial intelligence [10-12], the former utilizes power quality monitors (PQMs) for enhancements in the applicability to different types of TVDS caused by various reasons, the consistency of accuracy with different grid sizes and structures, and the simplicity without complicated training beforehand. 
There are two major tasks in the automatic location of the TVDS based on the graph theory and matrix algorithm. The first task is the direction-judgment at each PQM. It determines the relative positions between the TVDS and each PQM. In 2000, Parsons et al. first proposed the disturbance power (DP) algorithm and the disturbance energy (DE) algorithm for voltage-sag events [13]. Improved DE [14] and other algorithms based on different PQ disturbance features, e.g., instantaneous energy [15], instantaneous voltage and current [16], have been published for the direction-judgment of TVDS. However, there was a common weakness in the binary direction-judgments in these algorithms: the judgments only indicate the TVDS locating in the UP or DOWN area with respect to each PQM, which could be affected by many factors in a real distribution grid. However, there were no metric to evaluate the reliability or credibility of these results numerically. Additionally, in the smart distribution grid, an increasing number of distributed generators (DG) are being integrated, which has demonstrated critical influences on the existing direction-judgment algorithms $[17,18]$.

The second task in automatic location of the TVDS is to determine the location of the TVDS based on the direction-judgment in the whole NPQMS. Enabled by the theory of fault-location in power grids, the matrix-based approach was first proposed by Won et al. [8], which integrated all the direction-judgments and the topological information of the PQMs for the final location. Several studies have tried to improve its performance or reduced the implementation cost [9,19]; the concept of virtual PQM was also introduced [9] to handle incomplete PQM installation. However, these traditional matrix-based methods lack the fault-tolerant capability: if any direction misjudgment exists in the NPQMS, the location result of the TVDS would become incorrect. The accuracy of the final location result depends on the absolutely reliable direction-judgments at every PQM, which is hard to guarantee in practice. To address this issue, the disturbance measure (DM) utilized the disturbance intensity to acquire both the direction and quantity of the transient voltage disturbance signal [20], but the disturbance intensity is only one of many influence factors that need to be considered. In [17,21], the sensitivity and impact of the DG integration were considered, but the mechanism and rules behind the influence of DG remained to be further explained.

To locate the TVDS with better reliability and accuracy in the smart distribution grid, the following information can be utilized: (1) the credibility of direction-judgment corresponding to different influence factors should be integrated; (2) the influence of DG integration to direction-judgment should be verified and corrected; (3) multiple applicable criteria of direction-judgment for one same type of TVDS could be integrated as multi-evidences fusion; and (4) other important information, such as grid's topology, PQ measured data, layout of PQMs, and parameters of gird's components, among others, should be integrated for PQ information completing and correlating. Considering these multi-source information and evidences with different properties, the existing matrix-based methodologies for TVDS location cannot provide good performance. Information fusion (IF) techniques [22] can improve the decision-making process in an uncertain environment by the fused information with correlative and integrative properties.

In this paper, we first analyze the influence of the multiple factors to the reliability of the direction-judgment at each PQM in the NPQMS. Then, an improved fault-tolerant approach based on the IF techniques is proposed to locate the TVDS for DG integrated smart distribution grid. The main contributions of this paper are summarized as follows:

1. Three credibility factors are developed to denote quantitatively the reliability of the direction-judgment of TVDS at each PQM considering multiple influence factors, including the disturbance intensity and the fluctuation characteristic of transient voltage disturbance signals, and estimation errors at the virtual PQMs.

2. Two influence rules of DG integration on the direction-judgments are proposed. The rules can be used to verify whether mistake or reliability weakening takes place in direction-judgment at each PQM in typical scenarios. It is the basis to correct the fault location of TVDS caused by the DG integration in the subsequent decision-making. 
3. An improved fault-tolerant approach, based on the IF techniques is proposed to automatically locate the TVDS for DG integrated smart grid. Three critical schemes, including multi-credibilities fusion and multi-evidences fusion, credibility weakening of conflict points, and correction for DG integration, have been integrated for consideration in the proposed fusion method. Simulation results demonstrate that the proposed approach has significantly improved fault-tolerant capability and good applicability for various types of TVDS.

The rest of this paper is organized as follows. Section 2 introduces the limitation of the existing methods and the overall framework of the proposed approach. Section 3 provides the detailed analysis about the credibility factors that may affect the direction-judgments. Section 4 describes the decision-making strategy for the automatic location of the TVDS based on multi-evidence fusion theory, with consideration of the DG integration. Section 5 sets up simulation models and analyzes experimental results. Section 6 draws some conclusions.

\section{Overview of the Methodology}

\subsection{Limitation of Existing Matrix-Based Methods}

To automatically locate the TDVS in a distribution network, existing matrix-based methods $[5,8,9]$ first generate the coverage-matrix $C_{L \times N}$ and the direction-matrix $D_{N \times 1}$, where $L$ and $N$ are the total numbers of the lines and the monitors, respectively. $C_{L \times N}$ denotes the relative directions between each line and each PQM in the grid, and $D_{N \times 1}$ denotes the relative directions between the TVDS and each PQM when disturbance event happens. Their elements are illustrated in Equations (1) and (2). Then the result-matrix $R_{L \times 1}$ can be obtained by $R_{L \times 1}=\left[r_{1}, r_{2}, \ldots, r_{L}\right]^{T}=C_{L \times N} \times D_{N \times 1}$. If there exists $R_{j}=\max \left\{r_{1}, r_{2}, \ldots, r_{L}\right\}=N$, the line $L_{j}$ corresponding to $r_{j}$ is determined as the location of the TVDS.

$$
\begin{gathered}
c_{j i}=\left\{\begin{array}{lll}
+1, & \text { if } & L_{j} \text { is in DOWN-area of } \mathrm{PQM}_{i} \\
-1, & \text { if } & L_{j} \text { is in UP-area of } \mathrm{PQM}_{i}
\end{array}\right. \\
d_{i}=\left\{\begin{array}{lll}
+1, & \text { if } & \text { TVDS is in DOWN-area of } \mathrm{PQM}_{i} \\
-1, & \text { if } & \text { TVDS is in UP-area of } \mathrm{PQM}_{i}
\end{array}\right.
\end{gathered}
$$

Although the traditional matrix-based methods are fairly convenient to indicate the relative directions and locate the TVDS, there are several critical drawbacks which lead to the absence of the fault-tolerant capability:

- The signed binary integers (i.e., +1 or -1 ) of direction judgments cannot measure the reliability of results. Factors contributing to certain level of errors in the direction judging process include disturbance intensity, fluctuation characteristic of the PQ disturbance signals, data missing or errors in acquisition, and nodes without actual PQMs.

- The significant influences of DG integration on the direction-judgment are not considered, which may lead to an error in the location result of the TVDS in certain situations.

- The traditional matrix-based methods cannot comprehensively utilize multi-evidences and multi-credibilities in location algorithms for decision-making, especially when some direction-judgments are contradictory among different algorithms or incorrect due to DG integration.

To illustrate the limitation of the traditional matrix-based methods, Figure 1 shows the topology of a simple radial distribution system. Assuming that for cost considerations, there is no actual PQM installed at $L_{5}$ and $L_{9}$. Consider, as an example, a voltage-sag event occurs at $L_{5}$ and only one direction misjudgment takes place at either $M_{3}$ or $M_{6}$. Using the matrix-based method shown in Figure 2, the coverage-matrix $C$ and either of the two partially incorrect direction-matrixes $D$ will be generated. However, as shown in the result-matrixes $R$, in both cases the matrix-based method will fail to locate correctly the preset TVDS at $L_{5}$. 


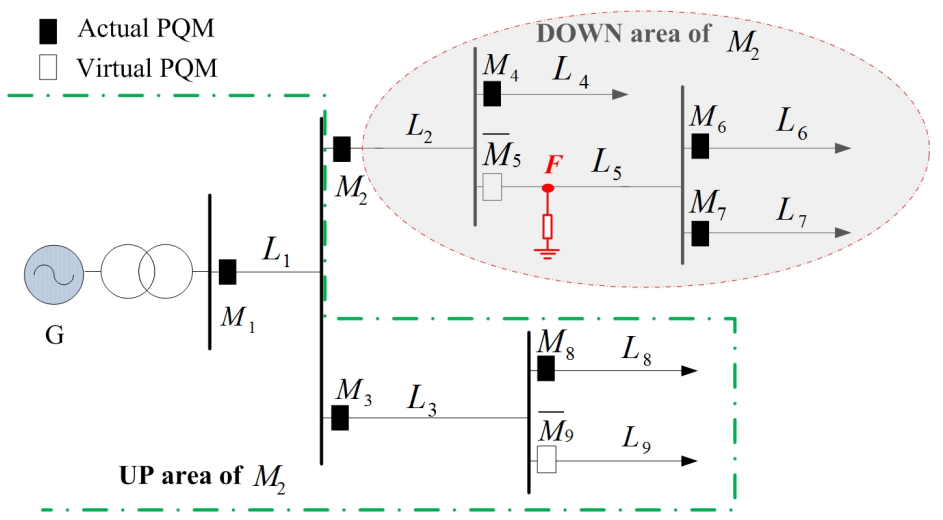

Figure 1. Topology of a simple grid and definition of DOWN/UP area.

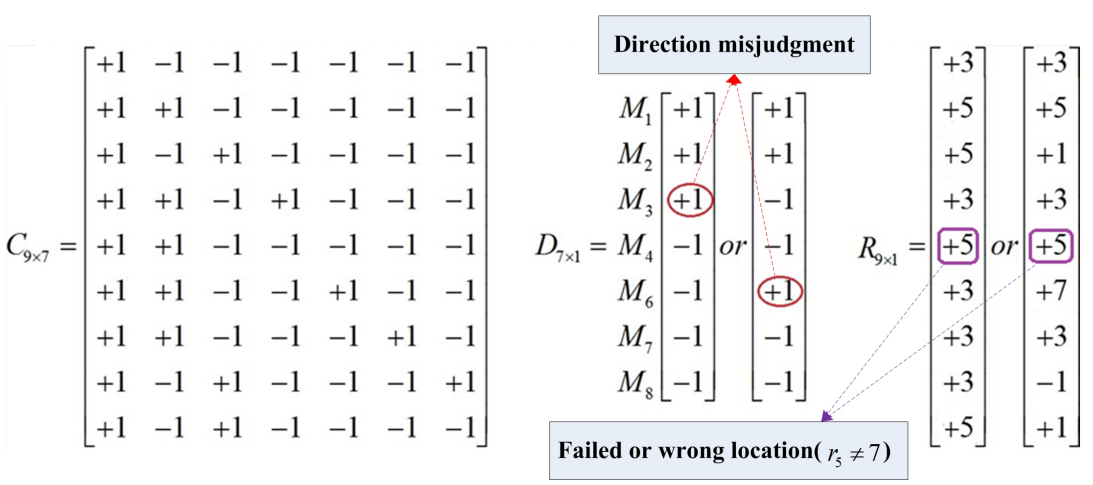

Figure 2. Failure in TVDS location with one direction misjudgment.

\subsection{Framework of the Proposed Approach}

An overview of the framework of the proposed fault-tolerant location approach for TVDS based on IF is shown in Figure 3. The preliminary modules in this framework during the pre-process are shown in white boxes, while the modules for the proposed approach are shown in orange boxes.

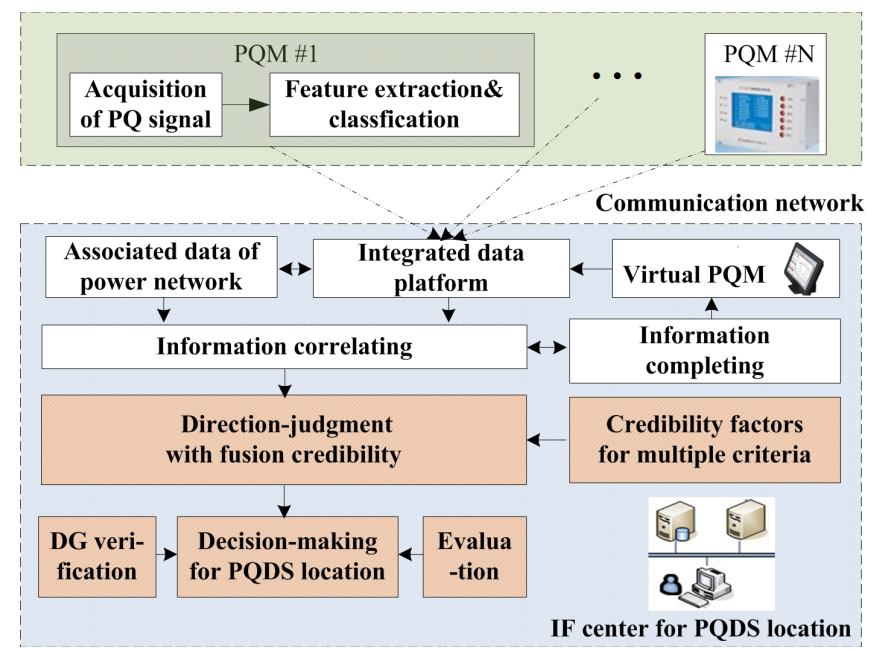

Figure 3. Overall framework of the proposed approach.

There are three basic components in the NPQMS, including all the monitors installed at the lines, the communication network and the IF center. First, the PQMs are responsible for disturbance PQ signals acquisition, features extraction, and disturbances identification. Then, the most important 
data-processing tasks, such as information correlating, information completing, and intelligent decision-making, will be executed in the IF center. Due to economic concerns and technical issues in practice, the PQ information in the NPQMS is often incomplete $[23,24]$, and the information completing module is thereby set up to solve this problem with the technique of PQ state estimation. As the data center of the NPQMS, the integrated data platform need to collect and store all kinds of data, such as the acquisition and analysis data from the PQMs, the topological structure and components parameters of the grid, the layout information of the PQMs, the complete data from state estimation, the process data and results of decision-making, etc.

After the pre-process, four key modules in the focus of this paper at the IF center are:

- Credibility factors module: to measure the reliability of direction-judgment at each PQM with consideration of multiple credibility factors, including disturbance intensity, fluctuation characteristic and state estimation.

- DG verification module: to analyze the influences of DG integration on the direction-judgment, deduce the influence rules, and define the verification factor.

- Fusion credibility module: to obtain the fusion credibility of the direction-judgments based on Dempster-Shafer evidence theory, with integrated considering of multi-credibilities fusion and multi-evidences fusion.

- Decision-making module: to automatically locate the TVDS for DG integrated smart grid with satisfying fault-tolerant capability.

Each of the four modules will be described in the sections below.

\section{Credibility of Direction-Judgement}

To transient voltage disturbance event, both the polarity of the initial peak of the DP and the polarity of the final DE value can be taken as the criteria of the direction-judgment between the TVDS and each PQM. The DP is the difference in the total instantaneous power and the steady-state instantaneous power, and the DE is the integral of the DP representing the change in energy flow through the PQM due to the disturbance. In this paper, we develop a new credibility metric of direction-judgment to properly measure the influences caused by those factors. The metric includes the relative direction between the TVDS and each PQM used in the existing matrix-based methods, and adds the credibility of direction-judgments in various cases. Taking two typical criteria, i.e., the DP and DE algorithms, for example, three credibility factors are defined as follows. The following factors are valid to both voltage sag and voltage swell caused by all kinds of TVDS.

\subsection{Credibility Factor for Disturbance Intensity}

Voltage disturbance signals are the basis of all direction-judgment algorithms for the TVDS. In general, the disturbance signals at PQMs that are distant from the TVDS are relatively weaker due to line loss and bypass flow. The DG integration can also alter the intensity in certain subareas. With the same noise condition, the direction misjudgment is also more likely to occur under the weaker transient voltage disturbance signals. It is the major factor that affects the credibility of the direction-judgment.

Suppose that a voltage-sag event caused by a single-phase grounding fault occurs at $L_{6}$ in Figure 1, where a Gaussian white noise (GWN) with the same intensity is added. As shown in Figure 4, the impacts of the GWN on the direction-judgment are different at $M_{4}$ and $M_{8}$. Figure $4 \mathrm{a}, \mathrm{b}$ show that only the disturbance signals be changed slightly at $M_{4}$, but the direction-judgment is not affected. Meanwhile, the PQ disturbance intensity at $M_{8}$ was quite weak, and thus we observed the misjudgment caused by this noise as shown in Figure 4c,d. 


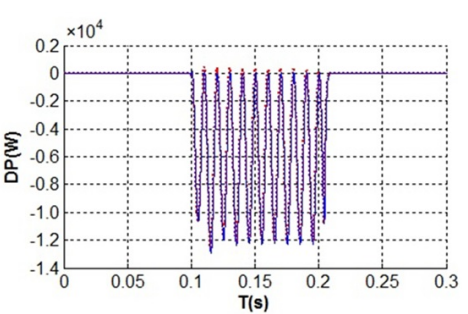

(a)

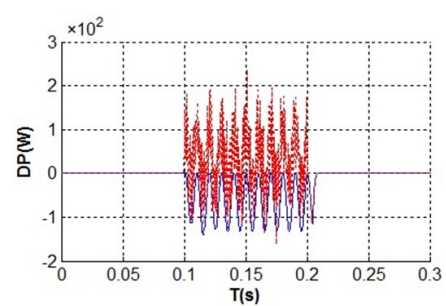

(c)

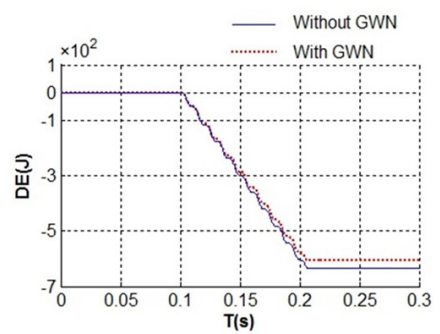

(b)

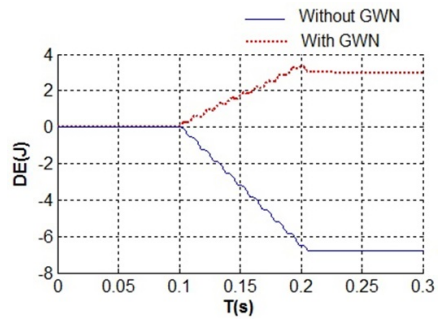

(d)

Figure 4. Effects on direction-judgments with different disturbance intensities. (a) DP at $M_{4} ;$ (b) DE at $M_{4} ;$ (c) DP at $M_{8} ;$ (d) DE at $M_{8}$.

To measure the effect of the transient voltage disturbance intensity with consideration to the two direction-judgment algorithms (DP and DE) $[13,14]$, the credibility factor $\alpha_{v, i}$ of disturbance intensity is defined as:

$$
\alpha_{v, i}= \begin{cases}1, & \text { if } k_{v, i}>\theta_{1} \\ \sqrt{k_{v, i}}+\left(1-\sqrt{\theta_{1}}\right), & \text { if } 0 \leq k_{v, i} \leq \theta_{1}\end{cases}
$$

in which

$$
k_{v, i}=\left|\Delta F_{v}(i)\right| /\left|\Delta F_{v, \max }\right|
$$

where $v$ is a criteria indicator: $v=1$ is for the DP algorithm and $v=2$ is for the DE algorithm. $\theta_{1}$ is a threshold value less than 1, and the two square roots in Equation (4) prevent $\alpha_{i}$ from being too small and transform the piecewise function to be a continuous function, respectively. $\Delta F_{v}(i)$ is the characteristic value of the transient voltage disturbance signal at $P Q M_{i}$ and $\Delta F_{v, \text { max }}$ is the maximum among all $\Delta F_{v}(i) . \Delta F_{1}(i)$ is the initial peak value of the DP signal and $\Delta F_{2}(i)$ is the stable final value of the DE signal. As shown in Figure $5 a$, the range of $\alpha_{v, i}$ is set as $\left(1-\sqrt{\theta_{1}}\right) \leq \alpha_{v, i} \leq 1$, and there is a strong positive correlation between $\alpha_{v, i}$ and $k_{v, i}$.

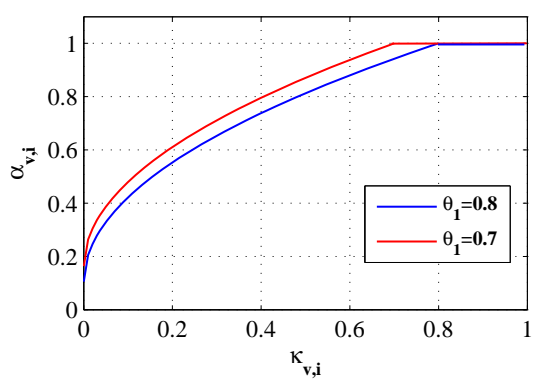

(a)

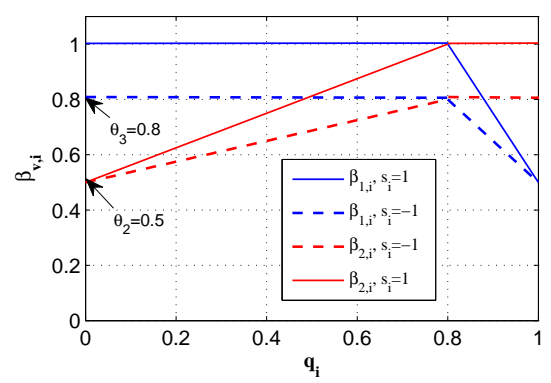

(b)

Figure 5. Distribution characteristics $\alpha_{v, i}$ of and $\beta_{v, i}$. (a) $\alpha_{v, i}$ with $\theta_{1}=0.8$ or $\theta_{1}=0.7$; (b) $\beta_{v, i}$ with $\theta_{2}=0.5$ and $\theta_{3}=0.8$. 


\subsection{Credibility Factor for Fluctuation Characteristics}

The fluctuation characteristics of the transient voltage disturbance signals could differ significantly, which shall be taken into consideration when calculating the possibility of direction misjudgment. Compared to the DP signal which yields more intense fluctuation, the DE signal is more stable in the presence of a voltage transient disturbance. According to [13], the threshold value, i.e., 0.8 , is the empirical preference between the two algorithms. Namely, if the final value of DE is greater than or equal to $80 \%$ of the peak excursion of DE during the event, then disturbance direction is determined by the DE algorithm; otherwise, the direction is determined by the DP algorithm.

The polarity fluctuation of the disturbance signal is also related to the credibility of direction-judgment. If the DP signal fluctuates frequently between the positive and negative polarities, the DE signal may fail to keep a strict monotonicity; the polarities between the final and initial peak DE values may even be reversed. In either case, its credibility should be reduced. As an example, Figure 6 shows the DE signals detected at $M_{7}$ and $M_{3}$ when a voltage-sag event takes place at $L_{7}$ in Figure 1 caused by capacitors switching. A direction misjudgment will occur at $M_{3}$ because of its distance from the TVDS that weakens the DE signal and leads to a failure of strict monotonicity.

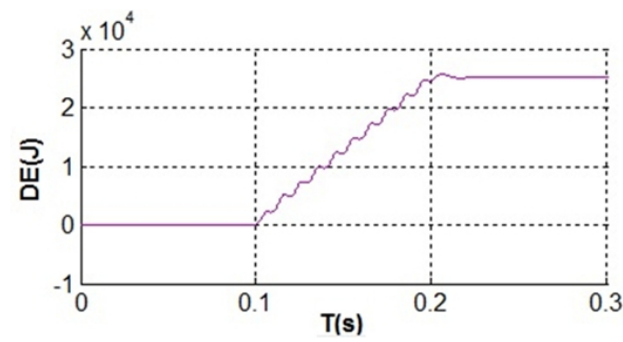

(a)

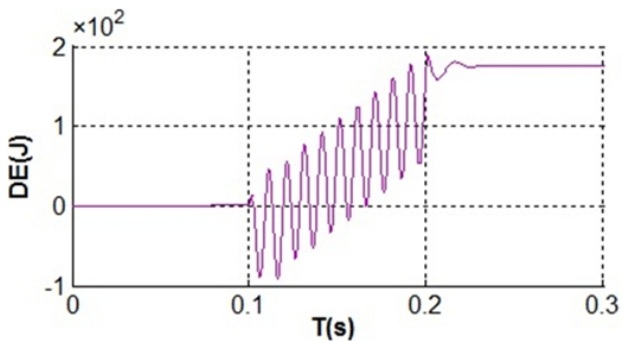

(b)

Figure 6. Effects on direction-judgments from different fluctuation characteristics. (a) DE signal at $M_{7}$; (b) DE signal at $M_{3}$.

According to the two laws for direction-judgment shown in [13], two new variables $q_{i}$ and $s_{i}$ can be defined to represent the characteristics of fluctuation intensity and monotonicity:

$$
\begin{gathered}
q_{i}=\left|\Delta E_{R}(i) / \Delta E_{P E}(i)\right| \\
s_{i}=\operatorname{sgn}\left[\Delta E_{I}(i) \cdot \Delta E_{R}(i)\right]
\end{gathered}
$$

where $\Delta E_{I}(i)$ and $\Delta E_{R}(i)$ are the initial peak value and final value of $D E$ signal at $P Q M_{i}$, respectively; $\triangle E_{P E}(i)$ is the peak excursion of DE signal during the PQ disturbance event at $P Q M_{i}$. If $q_{i} \geq 0.8$, the DE algorithm is more credible; otherwise, the DP algorithm is more credible. Meanwhile, if the polarity of the initial peak of the DP matches that of the final DE value (i.e., $s_{i}=1$ ), the direction-judgment should be assigned with higher credibility; otherwise, if $s_{i}=-1$, the credibility of direction-judgment should be reduced.

To measure the effect of fluctuation characteristics on the transient voltage disturbance signals, the corresponding credibility factors $\beta_{v, i}$ are defined as:

$$
\beta_{v, i}=\left[\beta_{1, i}, \beta_{2, i}\right]= \begin{cases}{\left[1-\frac{1-\theta_{2}}{0.2}\left(q_{i}-0.8\right), \quad 1\right],} & \text { if } q_{i} \geq 0.8 \cap s_{i}=1 \\ {\left[\theta_{3}-\frac{\theta_{3}-\theta_{2}}{0.2}\left(q_{i}-0.8\right), \quad \theta_{3}\right],} & \text { if } q_{i} \geq 0.8 \cap s_{i}=-1 \\ {\left[1, \frac{1-\theta_{2}}{0.8} q_{i}+\theta_{2}\right],} & \text { if } q_{i}<0.8 \cap s_{i}=1 \\ {\left[\theta_{3}, \frac{\theta_{3}-\theta_{2}}{0.8} q_{i}+\theta_{2}\right],} & \text { if } q_{i}<0.8 \cap s_{i}=-1\end{cases}
$$


where $\theta_{2}$ determines the minimum of $\beta_{v, i}$, and $\theta_{3}$ determines the maximum of $\beta_{v, i}$ when $s_{i}=-1$. If $s_{i}=1, \beta_{v, i} \in\left[\theta_{2}, 1\right]$; if $s_{i}=-1, \beta_{v, i} \in\left[\theta_{2}, \theta_{3}\right]$. Considering that the fluctuation characteristics of the voltage disturbance signal is also a factor in the credibility of the direction-judgment, we have refined the range of $\theta_{2}$ as $\theta_{2} \in[0.4,0.6]$ and $\theta_{2}<\theta_{3}<1$. Figure $5 \mathrm{~b}$ shows the general distribution characteristics of $\beta_{1, i}$ and $\beta_{2, i}$ in the case that we set $\theta_{2}=0.5$ and $\theta_{3}=0.8$.

\subsection{Credibility Factor for State Estimation}

To obtain the credibility factors $\alpha_{v, i}$ and $\beta_{v, i}$ at each node, the minimal requirement of actual PQM installment is to satisfy the complete observability of the power quality information in the NPQMS. With the optimal placement of the PQMs [19], the missing or erroneous PQ information can be completed reasonably with data from state estimation [23,24] and taken as data collected from a "virtual" PQM, such as $\bar{M}_{5}$ and $\bar{M}_{9}$ shown in Figure 1 . However, due to the estimation errors, the credibility of direction-judgments at the virtual PQMs should be reduced. Therefore, the credibility of state estimation $\chi_{v, i}$ is adopted as an additional factor to the major credibility factor $\alpha_{v, i}$.

According to the theory of uncertainty in measurement [25], the concept uncertainty of estimation is defined to reflect the possible distribution range of the estimation error and can be considered as the error limit under certain confidence probability. A relative deviation index $d_{i}$ can be defined as:

$$
d_{i}=\frac{h_{i, z_{1}}\left(x_{1}\right)-h_{i, z_{2}}\left(x_{2}\right)}{U_{i}(p)}
$$

where $U_{i}(p)$ is the expanded uncertainty with the confidence probability $p$ at virtual monitor $\bar{M}_{i}$. $x_{1}$ and $x_{2}$ are the state estimation results for one same state variable at $\bar{M}_{i}$ based on two different actual measuring points $z_{1}$ and $z_{2}$, and $h_{i, z_{1}}\left(x_{1}\right)$ and $h_{i, z_{2}}\left(x_{2}\right)$ are their corresponding measurement functions.

A larger $d_{i}$ means larger deviation between the two estimation results, and thus a lower credibility should be assigned. $\bar{M}_{i}$ can be considered as a normal estimation point if $\left|d_{i}\right| \leq 1$, or an abnormal estimation point if $\left|d_{i}\right| \geq \lambda$, or suspected estimation point if $1<\left|d_{i}\right|<\lambda$.

A feasible credibility function should satisfy the following requirements: the function should be continuous and smooth; if $\left|d_{i}\right| \leq 1$, the credibility of the state estimation at $\bar{M}_{i}$ should become saturated and close to 1 ; if $\left|d_{i}\right| \geq \lambda$, the credibility of the state estimation at $\bar{M}_{i}$ should be close to 0 ; if $1<\left|d_{i}\right|<\lambda$, the credibility should decrease sharply.

To measure the effect of the state estimation, the credibility factor $\chi_{v, i}$ is defined as:

$$
\chi_{v, i}=\left\{\begin{array}{ccc}
1, & \text { if } & \rho_{i}=0 \\
\max \left\{f\left(d_{i}\right), 0.5\right\}, & \text { if } & \rho_{i}=1
\end{array}\right.
$$

in which

$$
\begin{gathered}
f\left(d_{i}\right)=1-\left[g\left(d_{i}\right)+g\left(-d_{i}\right)\right] \\
g\left(d_{i}\right)=\frac{1}{1+e^{\frac{2 k}{\lambda}}\left[d_{i}+\left(1+\frac{\lambda}{2}\right)\right]}
\end{gathered}
$$

where $P Q M_{i}$ is an actual measuring point if $\rho_{i}=0$ and a virtual measuring point if $\rho_{i}=1$. According to [25], $k$ is a real number between 2 to 5 , and $\lambda$ is a natural number between 2 to 6 .

Figure 7 shows the distribution characters of $f\left(d_{i}\right)$ with different $\lambda$ and $k$. The drop of $f\left(d_{i}\right)$ is mainly determined by the parameter choice of $\lambda$. When $\lambda$ is fixed, the credibility values at the middle point, i.e., $\left|d_{i}\right|=2$, are the same despite different values of $k$. Meanwhile, the credibility values at another critical point, i.e., $\left|d_{i}\right|=1$, is significantly influenced by different $k$. Considering the requirements of the credibility function and the distribution characters of $f\left(d_{i}\right)$, this paper considers the choice of $\lambda=2$ and $k=3$. We also have $\chi_{1, i}=\chi_{2, i}$ where $\chi_{v, i}$ in the range of $\chi_{v, i} \in[0.5,1]$. 


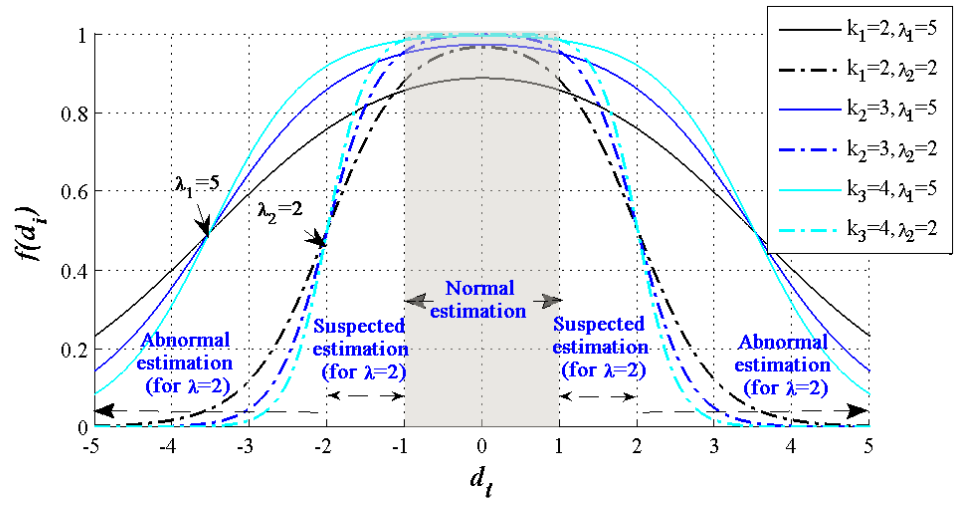

Figure 7. Curves of $f\left(d_{i}\right)$ with different $\lambda$ and $k$.

\section{DG Verification of Direction-Judgement}

In the smart distribution grid with DG integration, a traditional radial network with single power supply turns into a multi-source system, causing a significant variation in the power flow distribution [26,27]. Thus, the transient voltage disturbance intensity at each PQM may be affected, and direction misjudgments can take place in certain situation. Although the voltage sag events are used to demonstrate the influence of DG integration on TVDS, it is worth noting that the following analysis procedure and rules can also be applied to voltage swell caused by energy injection by simply reversing the polarity of the parameters.

\subsection{Influence Rules of DG Integration}

To analyze the general influence of the DG integration, we first transform the grid shown in Figure 1 to a scalable topology as shown in Figure 8. The partial networks $N_{a} \sim N_{d}$ represent any sub-networks with tree structure, which makes this model scalable for grids with radial structure. Moreover, we assume that one DG is connected to the grid at $B_{4}$ and a voltage-sag event $F$ takes place at $L_{1}$.

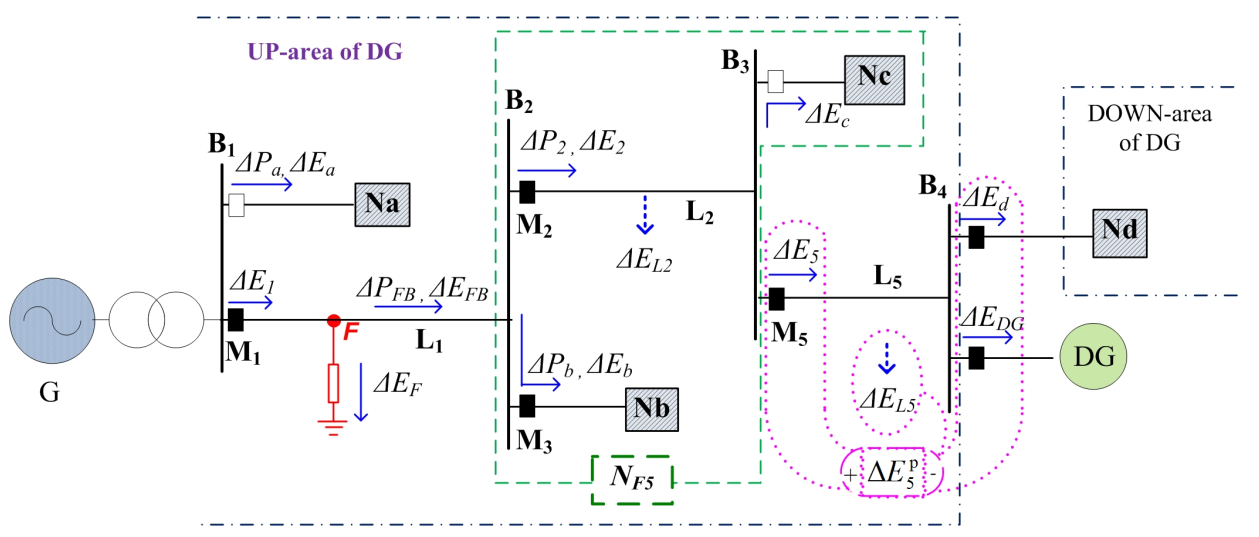

Figure 8. Scalable grid model with DG integration.

In Figure 8, we introduced the following notions: $(1)\left(A \_B\right)$ denotes the path from arbitrary component $\mathrm{A}$ to $\mathrm{B}$ in the grid; (2) $\operatorname{Link}_{(G-D G)}$ is the link between $\mathrm{G}$ and $\mathrm{DG}$, i.e., $\left(G_{-} L_{1} L_{2} L_{5} D G\right)$; (3) $\operatorname{Link}_{(F-D G)}$ is the link between DG and TVDS, i.e., $\left(F_{-} L_{2} L_{5} \_D G\right)$; $(4) B u s_{(F-D G)}$ is the bus connected to TVDS at the side of DG; and (5) $N_{F i}$ is the current-dividing network that includes all the partial networks and lines between $B u s_{(F-D G)}$ and $P Q M_{i}$. In addition, $\Delta P_{i}$ and $\Delta E_{i}$ are the $\mathrm{DP}$ and $\mathrm{DE}$ values at $P Q M_{i}, \Delta U_{F}$ and $\Delta U_{B 2}$ are the voltage variations at $F$ and bus $B 2$ in Figure 8 
during the voltage-sag event, $\Delta E_{F}$ is the DE value at TVDS, $\Delta P_{F B}$ and $\Delta E_{F B}$ are the DP and DE values transmitted from TVDS to $B u s_{(F-D G)}$, and $\Delta P_{F i}$ and $\Delta E_{F i}$ are the DP and DE values absorbed by $N_{F i}$. For the scalable topology shown in Figure 8, we can further distinguish it into different scenarios:

- Scenario 1: $\mathrm{F}$ is on $\operatorname{Link}_{(G-D G)}, P Q M_{i}$ is on $\operatorname{Link}_{(F-D G)},\left|\Delta P_{F i}\right|>\left|\Delta P_{F B}\right|$, and $\left|\Delta E_{F i}\right|>\left|\Delta E_{F B}\right|$.

- Scenario 2: F is on $\operatorname{Link}_{(G-D G)}, P Q M_{i}$ is on $\operatorname{Link}_{(F-D G)}$, but $\left|\Delta P_{F i}\right|<\left|\Delta P_{F B}\right|$ and $\left|\Delta E_{F i}\right|<\left|\Delta E_{F B}\right|$.

- Scenario 3: $\mathrm{F}$ is on $\operatorname{Link}_{(G-D G)}$, but $P Q M_{i}$ is not on $\operatorname{Link}_{(F-D G)}$.

- Scenario 4: F is not on $\operatorname{Link}_{(G-D G)}$, and $F$ is on the branch of $\operatorname{Link}_{(G-D G)}$ in the UP-area of DG.

- Scenario 5: F is not on $\operatorname{Link}_{(G-D G)}$, and $F$ is in the DOWN-area of DG.

(1) Scenario 1 and Scenario 2

In Scenario 1 and 2, since voltage sag is an energy-absorbing disturbance, we have $\Delta E_{F}>0$, $\Delta U_{F}<0$ and $\Delta U_{B 2}<0$. The power flow at $L_{1}$ could be positive or negative for the DG integration. But in either situation, we have $\Delta P_{b}<0, \Delta P_{F B}<0, \Delta E_{b}<0$ and $\Delta E_{F B}<0$. Based on the energy conservation law at $B_{2}$, we can obtain $\Delta P_{2}=\Delta P_{F B}-\Delta P_{b}$ and $\Delta E_{2}=\Delta E_{F B}-\Delta E_{b}$. Given that $N_{b}$ is actually $N_{F 2}$, two cases of the DP and DE algorithms of direction-judgment at $M_{2}$ can be deduced as:

$$
\begin{aligned}
& \Delta P_{2}=\left\{\begin{array}{llll}
>0 & (\text { DOWN-area }), & \text { if } & \left|\Delta P_{F 2}\right|>\left|\Delta P_{F B}\right| \\
<0 & (U P-\text { area }), & \text { if } & \left|\Delta P_{F 2}\right|<\left|\Delta P_{F B}\right|
\end{array}\right. \\
& \Delta E_{2}=\left\{\begin{array}{llll}
>0 & (\text { DOWN-area }), & \text { if } & \left|\Delta E_{F 2}\right|>\left|\Delta E_{F B}\right| \\
<0 & (\text { UP-area }), & \text { if } & \left|\Delta E_{F 2}\right|<\left|\Delta E_{F B}\right|
\end{array}\right.
\end{aligned}
$$

The direction-judgment at $M_{2}$ will be incorrect if $\left|\Delta P_{F 2}\right|>\left|\Delta P_{F B}\right|$ or $\left|\Delta E_{F 2}\right|>\left|\Delta E_{F B}\right|$. The same observation applies for the result obtained at $B_{3}$, and two possible direction-judgments at $M_{5}$ can be obtained according to the components of $N_{F 5}$ shown in Figure 8 . The direction-judgment at $M_{5}$ will be wrong if $\left|\Delta P_{F 5}\right|>\left|\Delta P_{F B}\right|$ or $\left|\Delta E_{F 5}\right|>\left|\Delta E_{F B}\right|$.

The scalable topology shown in Figure 8 can represent arbitrary distribution grid with a tree topology and single-DG integration. Subsequently, we can conclude the first influence rule of DG integration in direction-judgment as follows:

First influence rule: the direction-judgment at $P Q M_{i}$ is wrong and should be reversed if the TVDS is on $\operatorname{Link}_{(G-D G)}, P Q M_{i}$ is on $\operatorname{Link}_{(F-D G)}$, and $\left|\Delta P_{F i}\right|>\left|\Delta P_{F B}\right|$ for DP signal or $\left|\Delta E_{F i}\right|>\left|\Delta E_{F B}\right|$ for DE signal.

In the following discussion of Scenario 3 to 5, we will use the DE algorithm as an example to analyze the influence of DG integration for the direction-judgment.

(2) Scenario 3

In Scenario 3, there exist $\Delta E_{F}>0, \Delta U_{B 1}<0, \Delta U_{B 2}<0, \Delta U_{B 3}<0$, and $\Delta U_{B 4}<0$ when the voltage-sag occurs at $F$. Considering the effect of voltage stability of the DG integration, there also exist $\left|\Delta U_{B 1}\right|<\left|\Delta U_{B 1}^{\prime}\right|,\left|\Delta U_{B 2}\right|<\left|\Delta U_{B 2}^{\prime}\right|,\left|\Delta U_{B 3}\right|<\left|\Delta U_{B 3}^{\prime}\right|$ and $\left|\Delta U_{B 4}\right|<\left|\Delta U_{B 4}^{\prime}\right|$, where $\Delta U_{B 1}^{\prime}, \Delta U_{B 2}^{\prime}$, $\Delta U_{B 3}^{\prime}$ and $\Delta U_{B 4}^{\prime}$ are the variable values of voltage at the buses $B_{1}, B_{2}, B_{3}$ and $B_{4}$ if there has no DG integration, respectively. Except for the PQMs on $\operatorname{Link}_{(G-D G)}$, the variations of the DE values of the PQMs in the UP-area of TVDS (e.g., $\left.M_{1}, \bar{M}_{a}\right)$, on the branch of $\operatorname{Link}_{(F-D G)}$ (e.g., $M_{3}, \bar{M}_{c}$ ) and in the DOWN-area of DG (e.g., $M_{d}$ ), can be summarized as:

$$
\begin{cases}0<\Delta E_{1}<\Delta E_{1}^{\prime} & (\text { DOWN - area }) \\ \Delta E_{a}^{\prime}<\Delta E_{a}<0 & (U P-\text { area }) \\ \Delta E_{3}^{\prime}<\Delta E_{3}<0 & (U P-\text { area }) \\ \Delta E_{c}^{\prime}<\Delta E_{c}<0 & (U P-\text { area }) \\ \Delta E_{d}^{\prime}<\Delta E_{d}<0 & (U P-\text { area })\end{cases}
$$

where $\Delta E_{1}^{\prime}, \Delta E_{a}^{\prime}, \Delta E_{3}^{\prime}, \Delta E_{c}^{\prime}$ and $\Delta E_{d}^{\prime}$ are the variations of the $D E$ values at $M_{1}, \bar{M}_{a}, M_{3}, \bar{M}_{c}$ and $M_{d}$ if there has no DG integration, respectively. 
In Figure 8 , the given TVDS (i.e., $F$ ) is in the UP-area of $\bar{M}_{a}, M_{3}, \bar{M}_{c}$ and $M_{d}$, while in the DOWN-area of $M_{1}$. Therefore, the results of direction-judgment at all the PQMs except for those are on $\operatorname{Link}_{(G-D G)}$ are correct in this scenario, but only their disturbance intensities could be affected.

(3) Scenario 4 and Scenario 5

In Scenario 4, the simplified scalable topology of Figure 8 is shown in Figure 9a, where we have $\Delta E_{F}>0, \Delta U_{B 2}<0$ and $\Delta U_{B 4}<0$ when the voltage-sag occurs at $F$. Considering the effect of voltage stability of the DG integration, there also exist $\left|\Delta U_{B 2}\right|<\left|\Delta U_{B 2}^{\prime}\right|$ and $\left|\Delta U_{B 4}\right|<\left|\Delta U_{B 4}^{\prime}\right|$. The variations of the DE values at the PQMs in this scenario are:

$$
\begin{cases}0<\Delta E_{1}<\Delta E_{1}^{\prime} & (\text { DOWN-area }) \\ 0<\Delta E_{b}<\Delta E_{b}^{\prime} & \text { (DOWN-area }) \\ \Delta E_{2}^{\prime}<\Delta E_{2}<0 & \text { (UP-area }) \\ \Delta E_{d}^{\prime}<\Delta E_{d}<0 & \text { (UP-area })\end{cases}
$$

where $\Delta E_{b}^{\prime}, \Delta E_{2}^{\prime}$ are the variations of the DE values at $M_{b}$ and $M_{2}$ if there has no DG integration, respectively.

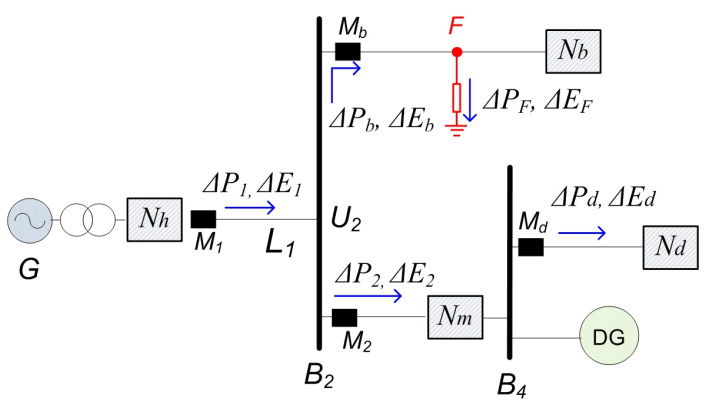

(a)

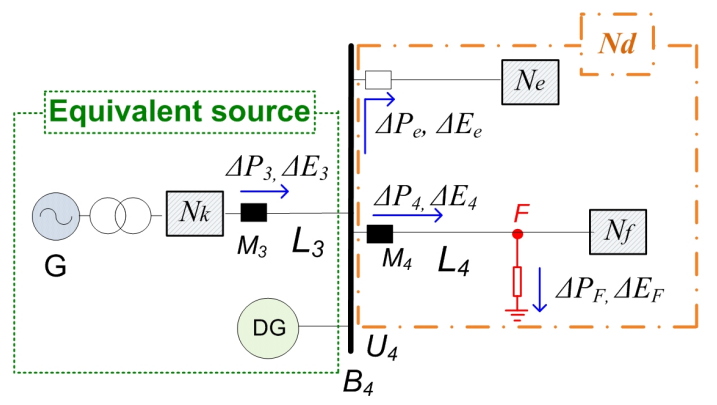

(b)

Figure 9. Simplified topologies in (a) Scenario 4 and (b) Scenario 5.

In Figure 9a, the given TVDS (i.e., $F$ ) is in the UP-area of $M_{2}$ and $M_{d}$, while in the DOWN-area of $M_{1}$ and $M_{b}$. Therefore, the results of direction-judgment at all the PQMs are correct in this scenario, but the disturbance intensities could be affected.

In Scenario 5, the simplified scalable topology is shown in Figure 9b. The partial network $N_{d}$ is expanded to two sub-networks (i.e., $N_{e}$ and $N_{f}$ ), and $G$ and DG can be regarded as one equivalent source. Similarly, we have $\left|\Delta U_{B 4}\right|<\left|\Delta U_{B 4}^{\prime}\right|$. The variations of the DE values at the PQMs in this scenario can be deduced as:

$$
\begin{cases}0<\Delta E_{3}<\Delta E_{3}^{\prime} & (\text { DOWN - area }) \\ \Delta E_{e}^{\prime}<\Delta E_{e}<0 & (\text { UP }- \text { area }) \\ 0<\Delta E_{4}<\Delta E_{4}^{\prime} & (\text { DOWN }- \text { area })\end{cases}
$$

where $\Delta E_{e}^{\prime}, \Delta E_{4}^{\prime}$ are the variations of the DE values at $\bar{M}_{e}$ and $M_{4}$ if there has no DG integration, respectively.

In Figure $9 b$, the given TVDS (i.e., F) is in the UP-area of $\bar{M}_{e}$ and the DOWN-area of $M_{3}$ and $M_{4}$. Therefore, the results of direction-judgment at all the PQMs are correct in this scenario, but the disturbance intensities could be affected.

Combining the analyses of Scenario 2, Scenario 3, Scenario 4, and Scenario 5, we can derive the second influence rule of DG integration in direction-judgment:

Second influence rule: in cases other than state in the first rule, only the intensity of transient voltage disturbance is influenced by the DG integration; the signs of the direction-judgments remain unaffected. 
Based on the above analysis, comprehensive simulation tests of the disturbance signals have been carried out to validate the two influence rules. Figures 10 and 11 illustrate the comparative case studies of Scenario 1. It has been demonstrated that the reversal of the direction-judgments agrees with the first influence rule of DG integration. As the other scenarios will not result in direction misjudgment, the figures of the disturbance signals are not shown herein.

- $\quad$ Case 1: $F$ is on $\operatorname{Link}_{(G-D G)}, P Q M_{i}$ is on $\operatorname{Link}_{(F-D G)},\left|\Delta P_{F i}\right|>\left|\Delta P_{F B}\right|$ and $\left|\Delta E_{F i}\right|>\left|\Delta E_{F B}\right|$.

- $\quad$ Case 2: same $F$ and $P Q M_{i}$ as Case 1 without DG integration.

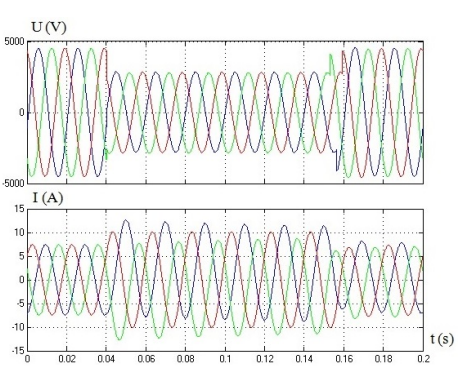

(a)

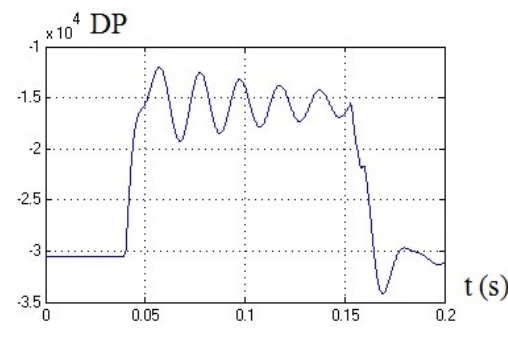

(b)

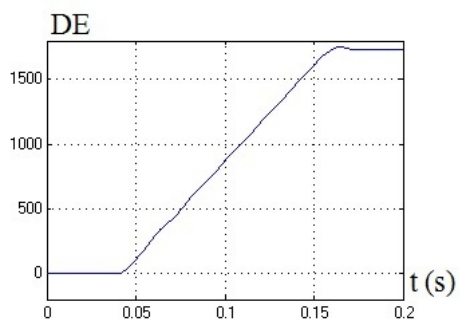

(c)

Figure 10. Disturbance signals for Case 1: (a) voltage and current at F; (b) DP signal (c) DE signal.

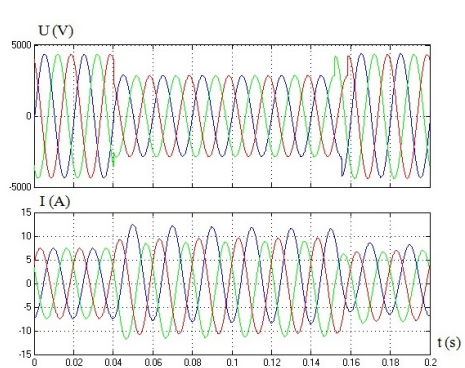

(a)

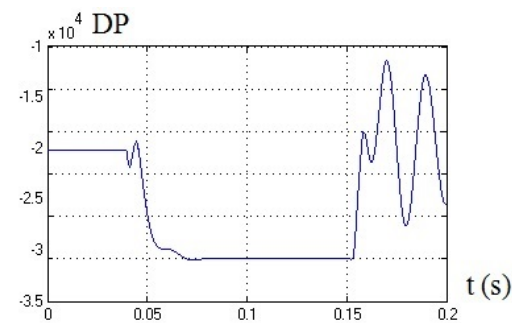

(b)

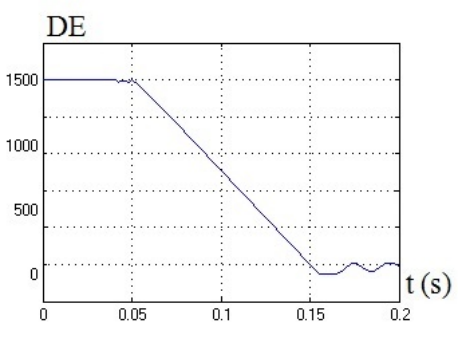

(c)

Figure 11. Disturbance signals for Case 2: (a) voltage and current at F; (b) DP signal; (c) DE signal.

It should be noted that a distribution grid with a circular topology or multiple DGs integration are more challenging due to the direction change of the power flow and the identification of the related links. While the proposed method is robust to different internal structures of the tree-like distribution grid and positions of the DG connected, further investigations should be conducted for more dynamic topology and DG configurations in practice.

\subsection{Verification Factor for DG Integration}

The first influence rule of DG integration cannot be directly utilized as the other factors because the TVDS location is unknown in advance. Instead, it can be used to verify the accuracy of the primary location result of the TVDS if the location is on $\operatorname{Link}_{(G-D G)}$. To achieve this goal, we define the partial-DE as follows:

$$
\Delta E_{j}^{\mathrm{p}}=\Delta E_{j}-\Delta E_{L j}-\Sigma \Delta E_{j}^{\text {out }}
$$

where $\Delta E_{L j}$ is the DE loss at $L_{j}, \sum \Delta E_{j}^{o u t}$ is the total DE value of all outgoing branches connected to the nearest bus at the DOWN-area of $L_{j}$. As an example, the components of $\Delta E_{5}^{p}$ at $L_{5}$ has been shown in Figure 8. 
By definition, $\Delta E_{j}^{p}=\Delta E_{F}$ if the TVDS is at $L_{j}$; otherwise, $\Delta E_{j}^{p}$ will be close to zero. Therefore, for any $L_{j}$ on $\operatorname{Link}_{(G-D G)}$, a partial verification factor $\delta_{j}$ is defined as:

$$
\delta_{j}=\left\{\begin{array}{l}
-1, \text { if } L_{j} \in \operatorname{Link}_{(G-D G)} \text { and } p_{j}=1 \\
+1, \text { otherwise }
\end{array}\right.
$$

in which

$$
p_{j}=\left|\Delta E_{j}^{p} / \Delta E_{\max }^{p}\right|
$$

where $\Delta E_{\text {max }}^{p}$ is the maximum among all $\Delta E_{j}^{p}$ on $\operatorname{Link}_{(G-D G)}$. If the primary location result is on $\operatorname{Link}_{(G-D G)}, \delta_{j}$ will be used for verification and correction, and only the line $L_{j}$ corresponding to $\delta_{j}=-1$ will be trusted as the actual TVDS.

\section{Location Based on Information Fusion}

\subsection{Credibility Fusion and Conflict Weakening}

The basic process of credibility fusion and conflict weakening is shown in Figure 12. The D-S evidence theory is adopted to realize the multi-credibilities fusion and the multi-evidences fusion. The details are introduced as follows.

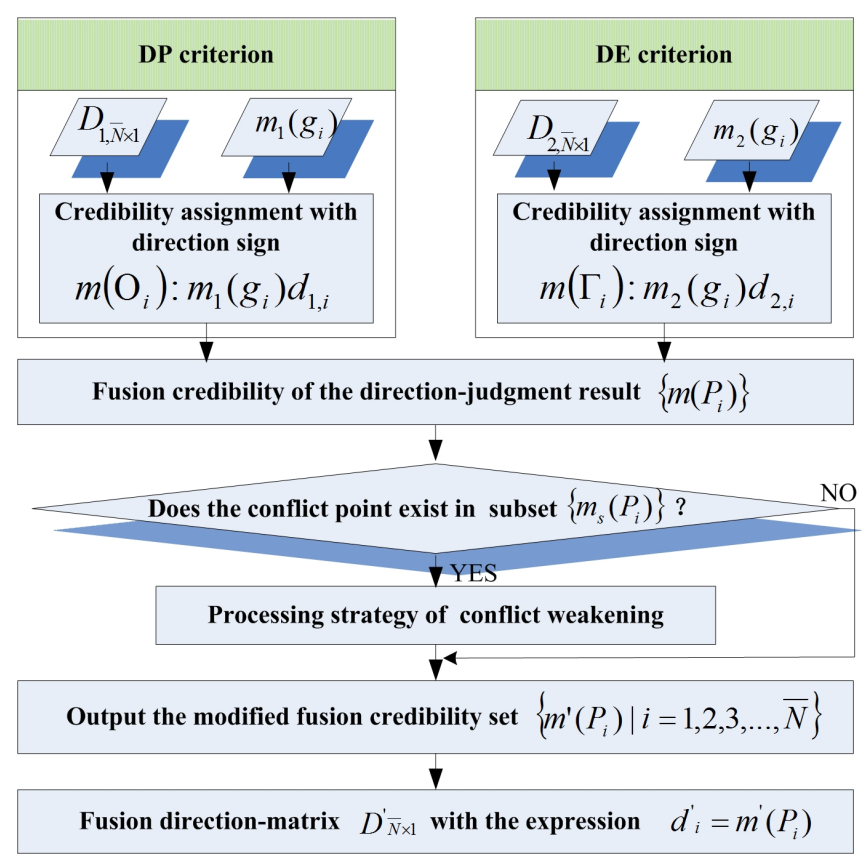

Figure 12. Basic process of credibility fusion with conflict weakening.

\subsubsection{Basic Probability Assignment Function}

In the D-S evidence theory, a discernment frame $\Theta$ defines a set that includes the basic focus elements corresponding to all of the possible decision results. For any focal element $P \subseteq \Theta$, the mass function $m(P)$ in Equation (17) can be taken as the prototype of the basic probability assignment function (BPAF).

$$
\left\{\begin{array}{l}
\sum_{P \subseteq \Theta} m(P)=1, \quad m(P) \geq 0 \\
m(\varnothing)=0
\end{array}\right.
$$


As for the decision goal of the TVDS location, supposing that each $P Q M_{i}$ in the NPQMS is numbered as $g_{i}$, we can construct $\Theta=\left\{g_{i} \mid i=1,2, \ldots, \bar{N}\right\}$, where $\bar{N}$ is the total number of all the actual and virtual PQMs.

Considering both the DP algorithm and the DE algorithm as the criteria of direction-judgment for the TVDS, their respective integrated credibility factor $\omega_{v}(i)$ of the direction-judgment at $P Q M_{i}$ can be obtained by:

$$
\omega_{v}(i)=\alpha_{v, i} \cdot \beta_{v, i} \cdot \chi_{v, i}
$$

where $v$ is the same criteria indication defined before (i.e. $v=1$ is for the DP algorithm and $v=2$ is for the DE algorithm).

The BPAF for both criteria are expressed as:

$$
m_{v}\left(g_{i}\right)= \begin{cases}\omega_{v}^{*}(i)=\omega_{v}(i) / \sum_{i=1}^{\bar{N}} \omega_{v}(i), & g_{i} \in \Theta \\ 0, & g_{i}=\varnothing\end{cases}
$$

\subsubsection{Credibility Fusion Based on D-S Evidence Theory}

For the TVDS location, the direction-judgments from the two criteria can be taken as two different evidences, and each of them has respective BPAF and complete direction-matrix $D_{v, \bar{N} \times 1} . D_{1, \bar{N} \times 1}$ and $D_{2, \bar{N} \times 1}$ can be generated by Equation (2) according to the DP algorithm and the DE algorithm, respectively. Therefore, two groups of basic credibilities containing both the direction-judgment information and the credibility information can be constructed as:

$$
\left\{\begin{array}{l}
m\left(O_{i}\right): m_{1}\left(g_{1}\right) d_{1,1}, \ldots, m_{1}\left(g_{\bar{N}}\right) d_{1, \bar{N}} \\
m\left(\Gamma_{i}\right): m_{2}\left(g_{1}\right) d_{2,1}, \ldots, m_{2}\left(g_{\bar{N}}\right) d_{2, \bar{N}}
\end{array}\right.
$$

where $O_{i}$ and $\Gamma_{i}$ are the focal elements of the two BPAF, $d_{1, i}$ and $d_{2, i}$ are the row elements of $D_{1, \bar{N} \times 1}$ and $D_{2, \bar{N} \times 1}$, respectively. $m_{1}\left(g_{i}\right)$ and $m_{2}\left(g_{i}\right)$ are the values of the BPAF corresponding to the two criteria.

D-S evidence theory can be used to obtain the fusion credibility of these two criteria. However, in some cases, the direction-judgments based on the two criteria at a given PQM may be conflict (i.e., $\left.d_{1, i} \neq d_{2, i}\right)$, in which the evidence combination rule of traditional D-S theory is unable to address evidence data with different signs. To solve this problem, an improved evidence combination rule is proposed:

$$
m\left(P_{i}\right)= \begin{cases}s_{1}\left(K^{\tau}\right)^{-1} \sum_{P_{i}=\mathrm{O}_{i} \cap \Gamma_{i}}\left|m\left(\mathrm{O}_{i}\right) m\left(\Gamma_{i}\right)\right|, & s_{1}=s_{2} \text { or } \quad\left\{s_{1} \neq s_{2}, k_{m} \geq 1\right\} \\ s_{2}\left(K^{\tau}\right)^{-1} \sum_{P_{i}=\mathrm{O}_{i} \cap \Gamma_{i}}\left|m\left(\mathrm{O}_{i}\right) m\left(\Gamma_{i}\right)\right|, & s_{1} \neq s_{2}, k_{m}<1 \\ 0, & P_{i}=\phi\end{cases}
$$

in which

$$
\begin{gathered}
K^{\tau}=1-K=1-\sum_{\mathrm{O}_{i} \cap \Gamma_{i}=\phi}\left|m\left(\mathrm{O}_{i}\right) m\left(\Gamma_{i}\right)\right| \\
s_{1}=\operatorname{sgn}\left[m\left(\mathrm{O}_{i}\right)\right], \quad s_{2}=\operatorname{sgn}\left[m\left(\Gamma_{i}\right)\right] \\
k_{m}=\left|m\left(\mathrm{O}_{i}\right)\right| /\left|m\left(\Gamma_{i}\right)\right|
\end{gathered}
$$

where $K \in[0,1)$ is the conflict factor between the two focal elements $O_{i}$ and $\Gamma_{i}$, and $P_{i}$ is the intersection of $O_{i}$ and $\Gamma_{i}$. In this way, the sign of the fusion credibility $m\left(P_{i}\right)$ is either $s_{1}$ or $s_{2}$ if the signs of $m\left(O_{i}\right)$ 
and $m\left(\Gamma_{i}\right)$ are consistent; otherwise, the sign of $m\left(P_{i}\right)$ is determined by the one with the greater absolute value.

\subsubsection{Strategy of Conflict Weakening}

In general, the multi-evidence fusion can obtain more comprehensive and reliable direction-judgments [28]. But once a conflict point of direction-judgment (i.e., $s_{1} \neq s_{2}$ ) with a strong credibility is observed at a PQM, it is necessary to reduce its fusion credibility to weaken its effect on the TVDS location. To this end, we propose a strategy of conflict weakening as follows:

- $\quad$ Step 1: Sort the set $M=\left\{m\left(P_{i}\right) \mid i=1,2, \ldots, \bar{N}\right\}$ by the absolute values $\left|m\left(P_{i}\right)\right|$ in descending order, and generate a new subset $M_{s} \subset M$ with the elements ranking in the top $30 \%$ of $M$.

- Step 2: Check all the values of $m\left(O_{i}\right)$ and $m\left(\Gamma_{i}\right)$ corresponding to the elements in $M_{s}$. If there exists any conflict points, mark their fusion credibility and proceed to Step 3; otherwise, jump to Step 4.

- Step 3: Reduce the marked fusion credibility by $20 \%$ and distribute the total reduced credibility to those monitors that are not conflict points in $\left\{m\left(P_{i}\right) \mid i=1,2, \ldots, \bar{N}\right\}$ with the principle of equipartition. Then go back to Step 1 and repeat.

- Step 4: Output the final set $M$ of the modified fusion credibility $\left\{m^{\prime}\left(P_{i}\right) \mid i=1,2, \ldots, \bar{N}\right\}$.

The parameter choices here (i.e., the $30 \%$ and $20 \%$ ) are determined empirically. According to the steps for the strategy of conflict weakening, if the parameter in Step 1 is set too large or the parameter in Step 3 is set too small, the efficiency of the strategy will be reduced. If the parameter in Step 1 is set too small, the effectiveness of the strategy is hard to be guaranteed, because some conflict points with relatively high credibility may not be selected into the subset $M_{s}$ for credibility weakening. If the parameter in Step 3 is set too large, the variation of the fusion credibility may be greater than expected. Therefore, the two parameter choices are given as a trade-off between efficiency and effectiveness of the conflict weakening. In practice, they can also be adjusted appropriately to adapt to different actual application scenarios.

\subsection{Decision-Making and DG Verification}

According to the modified fusion credibility $\left\{m^{\prime}\left(P_{i}\right) \mid i=1,2, \ldots, \bar{N}\right\}$, the fusion direction-matrix $D_{\bar{N} \times 1}^{\prime}$ can be constructed with $d_{i}^{\prime}=m^{\prime}\left(P_{i}\right)$. Similarly to the existing matrix-based location methods, the fusion result-matrix can be obtained as:

$$
R_{L \times 1}^{\prime}=C_{L \times \bar{N}} \cdot D_{\bar{N} \times 1}^{\prime}
$$

The basic process of the decision-making and verification of the TVDS location is shown in Figure 13. In the general case without DG integration, the line $L_{j}$ corresponding to the row element $r_{j}^{\prime}=\max \left\{r_{1}^{\prime}, r_{2}^{\prime}, \ldots, r_{L}^{\prime}\right\}$ is the location result of the TVDS. But due to the DG integration, this primary location result will be incorrect if $L_{j}$ is on $\operatorname{Link}_{(G-D G)}$.

According to the first rule of influence and the partial verification factor $\delta_{j}$ in Equation (15), we proposed the following verification and correction process to obtain the adjusted location result of the TVDS in the partial area of $\operatorname{Link}_{(G-D G)}$.

- Step 1: Calculate $\Delta E_{j}^{p}$ and $\delta_{j}$ for all lines on $\operatorname{Link}(G-D G)$.

- Step 2: If $\delta_{j}=+1$ for $L_{j}$ (the primary location result), go to Step 3; otherwise, let $\delta_{j^{\prime}}=\delta_{j}$ and jump to Step 4 .

- Step 3: Check all $\delta_{j}$ calculated in Step 1, and mark the one whose value equals to -1 as $\delta_{j^{\prime}}$.

- Step 4: Mark the line $L_{j^{\prime}}$ corresponding to $\delta_{j^{\prime}}$ as the adjusted location result of the TVDS. 


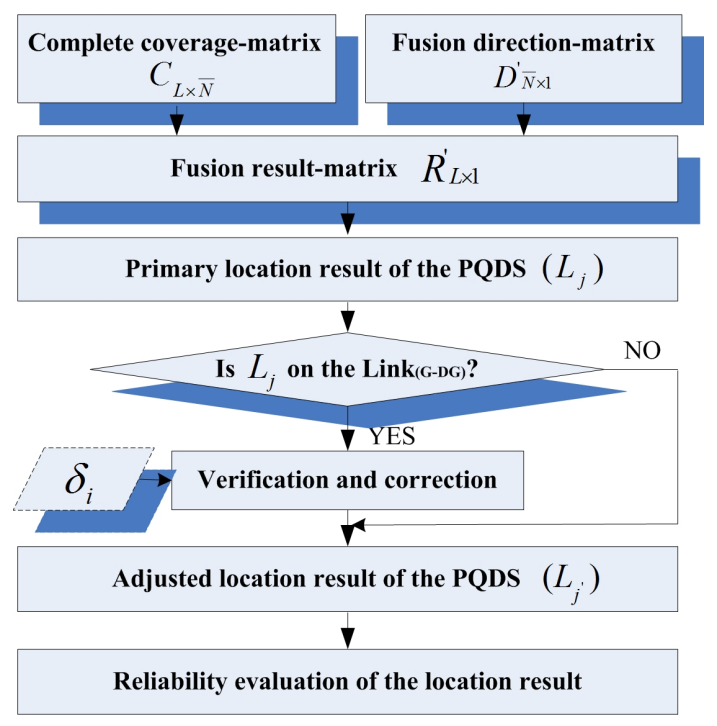

Figure 13. Basic process of decision-making and verification.

\subsection{Reliability Evaluation of Location Result}

To evaluate the reliability of the location result of the TVDS, we utilize the concordance index of multiple evidence sources [29] for the two groups of basic credibilities. Meanwhile, the errors and conflict points in direction-judgment shall reduce the reliability of the location result, especially when these points are on $\operatorname{Link}_{(G-F)}$, the link from G to TVDS and the branches in its DOWN-area. Based on the above considerations, the reliability coefficient $H$ is defined as:

$$
H=\exp \left\{\sum_{i=1}^{\bar{N}}\left[-\left(m_{1}\left(g_{i}\right)-m_{2}\left(g_{i}\right)\right)^{2}\right]\right\} \cdot\left[1-\frac{\sum_{i=1}^{\bar{N}} \rho_{i} \cdot n_{i}^{\prime}}{\bar{N}}\right]
$$

in which

$$
\begin{aligned}
& n_{i}^{\prime}=\left\{\begin{array}{ccc}
1.5, & \text { if } & P Q M_{i} \text { is a misjudgment point } \\
1, & \text { if } & P Q M_{i} \text { is a conflict point } \\
0, & \text { otherwise } &
\end{array}\right. \\
& \rho_{i}= \begin{cases}2, & \text { if } P Q M_{i} \text { is in the DOWN-area of } \operatorname{Link}_{(G-F)} \\
1.5, & \text { if } P Q M_{i} \text { is in the UP-area of } \operatorname{Link}_{(G-F)} \\
1, & \text { if } P Q M_{i} \text { is not on } \operatorname{Link}_{(G-F)}\end{cases}
\end{aligned}
$$

where $n_{i}^{\prime}$ is used to identify whether $P Q M_{i}$ is a direction misjudgment point or conflict point, and $\rho_{i}$ denotes the relative position of $P Q M_{i}$ and $\operatorname{Link}_{(G-F)}$. The values setting of $n_{i}^{\prime}$ and $\rho_{i}$ are done according to their relative impacts to the reliability of the location result. The value range of $H$ is $H \in\left(e^{-4}, 1\right)$, where a higher value of $H$ indicates a higher reliability.

\section{Case Study}

\subsection{Simulation Model Description}

Figure 14 shows the topology of an IEEE 13 node test feeder with a nominal voltage of $115 \mathrm{kV} / 4.16 \mathrm{kV}$ and a nominal power capacity of $5000 \mathrm{kVA}$ [30]. There are 18 lines in the system, numbered from $L_{1}$ to $L_{18}$. For the NPQMS, the monitors marked in Figure 14 include both 13 actual PQMs and 5 virtual PQMs $(\bar{N}=18)$. 


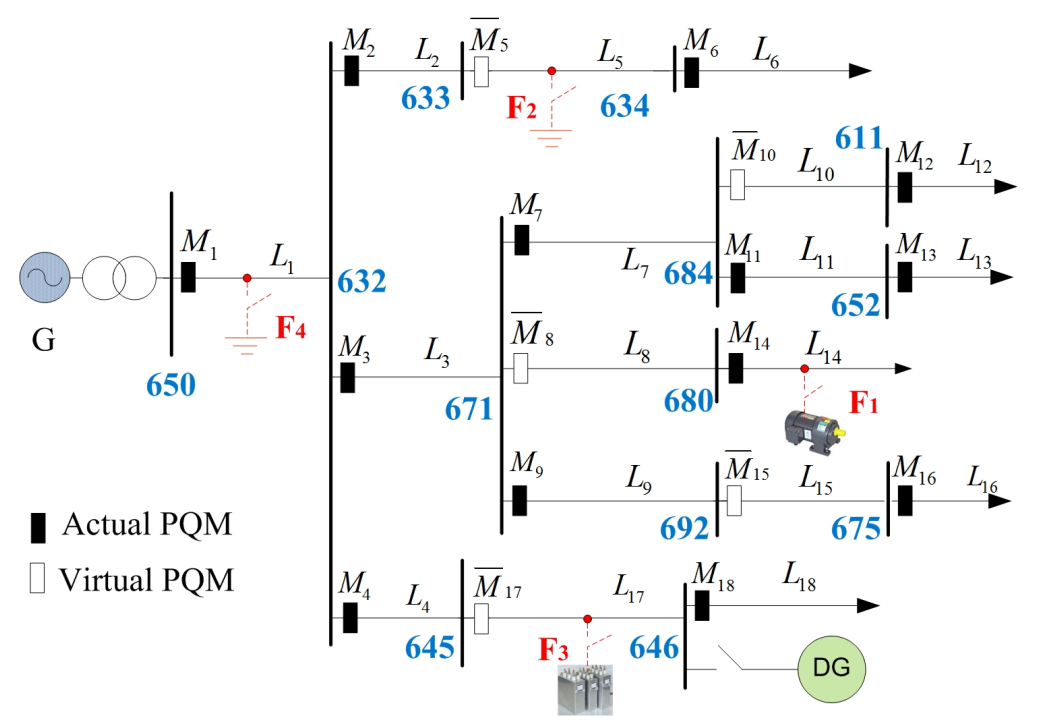

Figure 14. IEEE 13 node test feeder with DG integration.

To verify the effectiveness of the proposed approach, we use three settings to generate different cases of direction misjudgments at some PQMs as follows:

1. GWN with the mean value in range of $[-5 \%,+5 \%] P_{i}^{s s}$ are added to some monitoring points, where $P_{i}^{s S}$ is the steady-state active power at $P Q M_{i}$.

2. A DG with $10 \% S_{N}$ is connected to Node 646 , where $S_{N}$ is the nominal power capacity of the system.

3. Three different kinds of events are used to simulate the voltage-sag disturbance, including the line to ground fault, capacitor switching $(550 \mathrm{kVar})$ and starting of large-size induction motor $(37.3 \mathrm{~kW})$.

Four typical transient voltage disturbance events for tests, including their locations, causes and DG integration, are shown in Table 1. The threshold values in Equations (3), (5) and (11) are set as: $\theta_{1}=0.8, \theta_{2}=0.5, \theta_{3}=0.8, k=3$ and $\lambda=2$. The simulations are performed in MATLAB/simulink environment.

Table 1. Description of different transient voltage disturbance events.

\begin{tabular}{ccccc}
\hline Event & Event Location & Event Cause & DG Integration & Adding Points of GWN \\
\hline$F_{1}$ & $L_{14}$ & Starting of motor & No & $M_{7}, M_{11}, M_{13}$ \\
$F_{2}$ & $L_{5}$ & Line to ground & Yes & $M_{3}, M_{7}, M_{11}$ \\
$F_{3}$ & $L_{17}$ & Capacitor switching & Yes & $M_{3}, M_{7}, M_{11}$ \\
$F_{4}$ & $L_{1}$ & Line to ground & Yes & None \\
\hline
\end{tabular}

\subsection{Credibility Analysis}

With the PQ simulation data obtained at each PQM in the model, the complete direction-matrixes $D_{1, \bar{N} \times 1}$ and $D_{2, \bar{N} \times 1}$ can be generated by the DP algorithm and the DE algorithm, respectively. According to the analysis in Section 3, the three credibility factors $\alpha_{v, i}, \beta_{v, i}, \chi_{v, i}$ will be calculated. Then, according to the basic strategies of credibility analysis and fusion shown in Figure 12, all basic credibilities of the direction-judgments from both DP and DE algorithms and their fusion credibilities for the four disturbance events are calculated, as shown in Figure 15. Comparing the direction-judgments in these figures and the layout of all PQMs in Figure 14, the direction misjudgment points and conflict points for all these four disturbance events are shown in Table 2. 


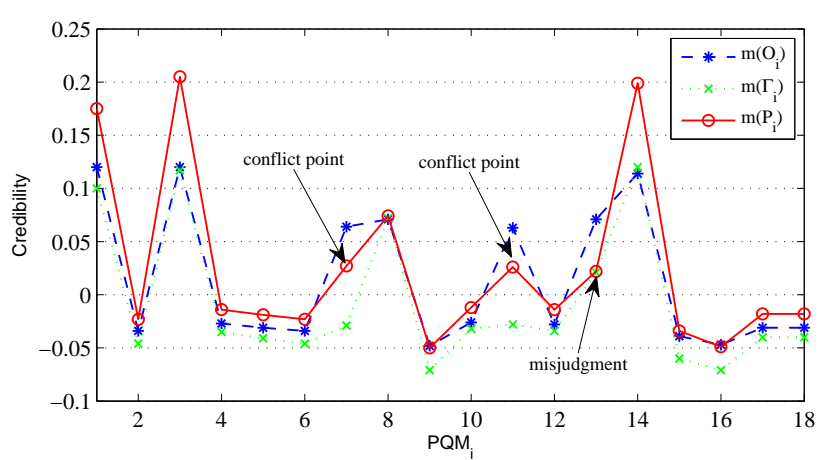

(a)

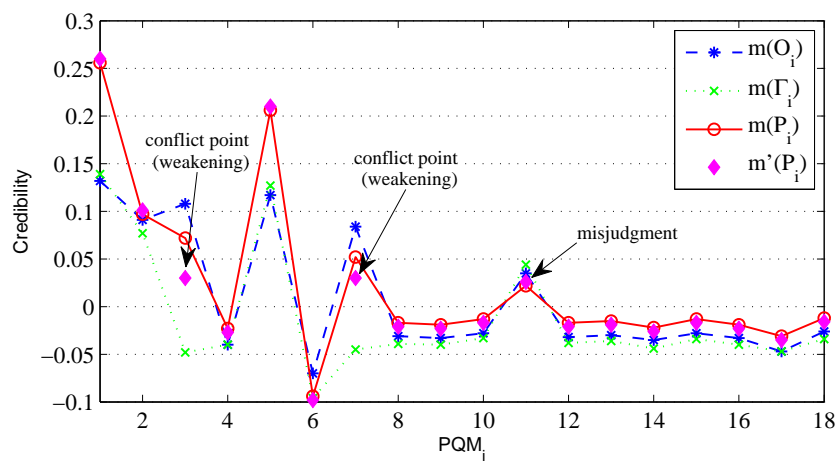

(b)

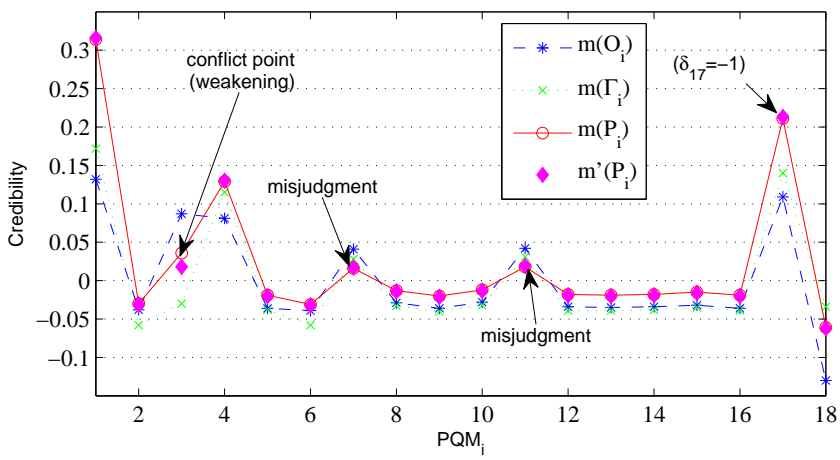

(c)

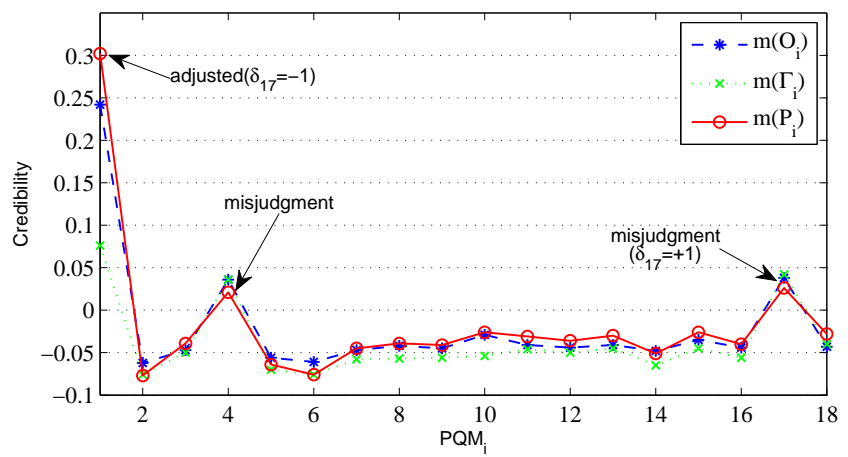

(d)

Figure 15. Distribution of basic credibilities and fusion credibilities for each disturbance event. (a) Event $F_{1} ;$ (b) Event $F_{2} ;$ (c) Event $F_{3}$; (d) Event $F_{4}$. 
Table 2. Direction misjudgments and conflict points.

\begin{tabular}{ccccc}
\hline Event & Direction Misjudgment & Conflict Point & Conflict Weakening & DG Verification \\
\hline$F_{1}$ & $M_{7}, M_{11}, M_{13}$ & $M_{7}, M_{11}$ & No & No \\
$F_{2}$ & $M_{3}, M_{7}, M_{11}$ & $M_{3}, M_{7}$ & Yes & No \\
$F_{3}$ & $M_{3}, M_{7}, M_{11}$ & $M_{3}$ & Yes & Yes \\
$F_{4}$ & $M_{4}, M_{17}$ & None & No & Yes \\
\hline
\end{tabular}

The conflict weakening was not activated because there is no conflict point in event $F_{4}$ and the two conflict points in event $F_{1}\left(M_{7}, M_{11}\right)$ are not in the top $30 \%$ of the sorted set $\left\{M\left(P_{i}\right) \mid i=1,2, \ldots, \bar{N}\right\}$; it was, however, activated in events $F_{2}$ and $F_{3}$ to reduce the fusion credibility of the conflict points. Taking the event $F_{2}$ for example, there were three direction misjudgment points in the whole NPQMS, and the two conflict points at $M_{3}$ and $M_{7}$ both activated the conflict weakening process as their fusion credibilities are in the top $30 \%$ of the sorted set. It is also notable that only a few non-conflict points with relatively larger basic credibilities by both criteria have noticeably increased fusion credibility; the fusion credibility on other measuring points were all lower than their basic credibilities. This means that, with the credibility fusion and conflict weakening, the location result of TVDS depends more on monitoring points with the same sign and relatively larger basic credibility according to the two criteria.

The configurations in Tables 1 and 2 have considered major factors for the TDVS location with fault tolerance, including different locations and causes of TVDS, settings of GWN and DG integration, and scenarios of direction misjudgment, conflict points and conflict weakening. Four events under testing are comprehensive combinations of these factors verify the effectiveness of the proposed methods in different scenarios.

\subsection{Decision-Making and Performance Comparison}

After the complete coverage-matrix $C_{L \times \bar{N}}$ and the fusion direction-matrix $D_{\bar{N} \times 1}^{\prime}$ are obtained, the location results of TVDS for each disturbance event will be determined. To demonstrate the fault-tolerant capacity of the IF-based location approach, we compared it with the traditional matrix-based (TM) methods [8,9] and the disturbance measuring (DM) algorithm [20]. The criteria and main features of them are shown in Table 3. All these three methods belong to the first location strategy that are based on the graph theory and matrix algorithm, and the proposed IF-based location approach can be viewed as an improved method of the other two methods.

Table 3. Criteria and features of different methods.

\begin{tabular}{cccc}
\hline Method & Criterion for TVDS Location & Credibility Factors & DG Integration \\
\hline TM & If $r_{j}=\max \left\{r_{1}, \ldots, r_{L}\right\}=\bar{N}, L_{j}$ is TVDS & Not considered & Not considered \\
DM & If $r_{j}=\max \left\{r_{1}, \ldots, r_{L}\right\}, L_{j}$ is TVDS & Partly consideration & Not considered \\
IF & If $r_{j}^{\prime}=\max \left\{r_{1}^{\prime}, \ldots, r_{L}^{\prime}\right\} \wedge \delta_{j^{\prime}}=-1, L_{j^{\prime}}$ is TVDS & Comprehensive consideration & Considered (two rules) \\
\hline
\end{tabular}

The location results are shown in Table 4. The location results showed that, for each case with direction misjudgment points, all the two existing matrix-based methods failed to locate the TVDS correctly. According to the criteria of TVDS location in the TM method, the TVDS cannot be determined if the maximum element in the result-matrix (i.e., $r_{j}=\max \left\{r_{1}, \ldots, r_{L}\right\}$ ) is unequal to $\bar{N}$. In contrast, the proposed IF-based approach predicted correctly in all cases, for its comprehensive consideration for the multi-information, such as multiple credibility factors, DG integration, multi-evidence, and etc. 
Table 4. Location Result of Different Methods.

\begin{tabular}{|c|c|c|c|c|c|c|}
\hline Event & Method & Max in $R$ or $R^{\prime}$ & TVDS & $\delta_{j}$ & $H$ & Result \\
\hline \multirow{3}{*}{$F_{1}$} & $\mathrm{TM}$ & $r_{14}=16$ & - & - & - & $\mathrm{F}$ \\
\hline & DM & $r_{13}=6.31$ & $L_{13}$ & - & - & $x$ \\
\hline & IF & $r_{14}^{\prime}=0.85$ & $L_{14}$ & - & 0.73 & $\sqrt{ }$ \\
\hline \multirow{3}{*}{$F_{2}$} & $\mathrm{TM}$ & $r_{5}=16$ & - & - & - & $\mathrm{F}$ \\
\hline & DM & $r_{11}=5.44$ & $L_{11}$ & - & - & $x$ \\
\hline & IF & $r_{5}^{\prime}=0.83$ & $L_{5}$ & - & 0.77 & $\sqrt{ }$ \\
\hline \multirow{3}{*}{$F_{3}$} & $\mathrm{TM}$ & $r_{18}=14$ & - & - & - & $\mathrm{F}$ \\
\hline & DM & $r_{11}=4.19$ & $L_{11}$ & - & - & $x$ \\
\hline & IF & $r_{17}^{\prime}=0.89$ & $L_{17}$ & -1 & 0.76 & $\sqrt{ }$ \\
\hline \multirow{3}{*}{$F_{4}$} & $\mathrm{TM}$ & $r_{17}=18$ & $L_{17}$ & - & - & $x$ \\
\hline & $\mathrm{DM}$ & $r_{17}=8.63$ & $L_{17}$ & - & - & $\times$ \\
\hline & IF & $r_{17}^{\prime}=1.0$ & Primary: $L_{17}$ & $\begin{array}{l}+1 \\
-1\end{array}$ & 0.71 & $\sqrt{ }$ \\
\hline
\end{tabular}

Note that the DG verification process was activated for the events $F_{3}$ and $F_{4}$, because both their primary location results were on $\operatorname{Link}_{(G-D G)}$. However, as shown in Figure 15c,d, the DG correction process was only activated for the event $F_{4}$, because only one partial verification factor $\delta_{17}$ among the primary location results was equal to +1 . It indicated that the primary location result $L_{17}$ in the event $F_{4}$ was incorrect because of the DG integration, and we could obtain the adjusted location result $L_{1}$ according to $\delta_{1}=-1$ on $\operatorname{Link}_{(G-D G)}$. The values of $H$ also showed the reliability for each location result of the TVDS. The relatively low reliability in event $F_{4}$ was due to all its misjudgment points , i.e., $M_{4}$ and $\bar{M}_{17}$, only taking place in the DOWN-area of $\operatorname{Link}_{(G-F)}$.

\subsection{Analysis of Fault Tolerance}

With different positions and causes of TVDS as well as settings of GWN and DG integration, we have set up three hundred tests in five groups according to the number of direction misjudgments. For each of these five groups, we compare the fault tolerance of the proposed IF approach with the TM algorithm and the DM algorithm. The results are shown in Table 5.

Table 5. Comparison of fault tolerance of three location approaches.

\begin{tabular}{cccccccc}
\hline \multirow{2}{*}{ Misjudgments } & \multirow{2}{*}{ Test Times } & \multicolumn{2}{c}{ TM } & \multicolumn{2}{c}{ DM } & \multicolumn{3}{c}{ Proposed IF } \\
\cline { 3 - 7 } & & Correct & \% & Correct & \% & Correct & \% \\
\hline 0 & 60 & 60 & 100 & 60 & 100 & 60 & 100 \\
1 & 60 & N/A & - & 53 & 88.3 & 58 & 96.7 \\
2 & 60 & N/A & - & 43 & 71.7 & 55 & 91.7 \\
3 & 60 & N/A & - & 38 & 63.3 & 54 & 90.0 \\
4 & 60 & N/A & - & 31 & 51.6 & 52 & 86.7 \\
Total & 300 & 60 & 20.0 & 225 & 75.0 & 279 & 93.0 \\
\hline
\end{tabular}

According to the above data, the performances of both the TM and the DM algorithms are far from satisfactory in the presence of direction misjudgments in the NPQMS. The TM algorithm only works with the absolute reliability of all direction-judgments. The DM algorithm locates the TVDS correctly in 165 of 240 tests with direction misjudgments (75\% accuracy on average). For the proposed IF approach, there are only 22 mistakes ( $93 \%$ accuracy on average). The proposed IF approach provides a remarkable improvement with fault-tolerant capability by taking into full account of different influence factors, multiple criteria of direction-judgment, and verification of DG integration.

Several factors may be related to the mis-location of TVDS in the proposed IF method, including:

- The number of direction misjudgments: more direction misjudgments lead to higher probability of location failure. 
- The position of the direction misjudgments: if direction misjudgments occur in the DOWN-area of the TVDS, it has higher probability of location failure.

- The credibility of the direction misjudgments: if credibility of the point with direction misjudgment is relatively high, it has higher probability of location failure.

- The relevancy of the direction misjudgments: strong relevance of the direction misjudgments, i.e., all misjudgments are located along the same link, leads to higher probability of location failure.

As shown in Table 6, two typical tests are provided to show the failure cases of TVDS location with the proposed IF approach. For event $F_{5}$, both the two direction misjudgments $M_{11}$ and $M_{13}$ are in the down-area of the TVDS (i.e., $L_{7}$ ). In event $F_{6}$, the number of direction misjudgments is relatively large (four misjudgments at $M_{3}, M_{4}, M_{17}$, and $M_{18}$ ), and there is strong relevance among $M_{4}, M_{17}$ and $M_{18}$ as they are located on the same link.

Table 6. Two typical tests of failure for TVDS location.

\begin{tabular}{ccccccccc}
\hline Event & Event Location & DG Integration & Direction Misjudgment & Conflict Point & DG Verification & TVDS & Result & $\boldsymbol{H}$ \\
\hline$F_{5}$ & $L_{7}$ & Yes & $M_{11}, M_{13}$ & - & - & $L_{13}$ & $\times$ & 0.614 \\
$F_{6}$ & $L_{9}$ & Yes & $M_{3}, M_{4}, M_{17}, M_{18}$ & - & - & $L_{18}$ & $\times$ & 0.611 \\
\hline \multicolumn{7}{c}{$\times$ Incorrect. }
\end{tabular}

Moreover, the probabilistic character of the location failure can be reflected in the reliability evaluation of location result, as shown in the latter part of Equation (26). The choices of parameter in Equations (27) and (28) partially underscore these probabilistic tendencies. By comparing the reliability coefficients $H$ in Tables 4 and 6 , the values of $H$ in the events $F_{5}$ and $F_{6}$ are significantly smaller, which means the location results for $F_{5}$ and $F_{6}$ have lower reliability.

\section{Conclusions}

In this paper, we proposed an information fusion based approach to TVDS location with fault-tolerant capacity in the DG integrated smart distribution grid. Based on the assumption of the complete observability of the power quality information in the NPQMS, the multi-credibility fusion algorithm integrates three influence factors and two criteria to quantify the reliability of the direction-judgment of TVDS at each PQM. Combined with the strategy of conflict weakening, the proposed IF-based approach improves the fault-tolerant capacity for TVDS location for distribution grids with a tree topology with and without DG integration. In the presence of DG integration, two influence rules and a correction algorithm have addressed the direction misjudgments caused by the DG integration at some PQMs, which significantly improved the fault-tolerance of TVDS location. Simulation results on the IEEE 13 node test feeder validated the effectiveness of the proposed approach. Compared with the existing matrix-based methods, the proposed approach holds the advantages of high accuracy and fault-tolerance capability under various influence factors.

The future research work aims to improve the generic applications of the IF-based location strategy of arbitrary topology and DG integration. In particular, theoretical foundation, simulation experiments and field tests will comprise the major tasks to developed the influence rules and correction strategy of DG integration for a circular topology and multiple DGs integration.

Acknowledgments: The research was supported by the Natural Science Foundation of China (Grant Nos. 51507153, 51207139), Zhejiang Provincial Natural Science Foundation of China (Grant No. LY17E070005), and the China Scholarship Council (Grant No. 201408330220).

Author Contributions: Guoqing Weng conceived and designed the algorithms and experiments; Feiteng Huang and Xiaodong Yang developed the simulation environment and performed the experiments; Jun Yan implemented the comparative studies and also implemented part of the simulation platform; Youbing Zhang and Haibo He analyzed the simulation results and comparative studies, and also led the overall research development for this work. All authors have contributed to the writing, editing, and proofreading of this paper.

Conflicts of Interest: The authors declare no conflict of interest. 


\section{Abbreviations}

The following abbreviations are used in this manuscript:

$\begin{array}{ll}\text { PQ } & \text { Power quality } \\ \text { PQM } & \text { Power quality monitor } \\ \text { NPQMS } & \text { Networked power quality monitoring system } \\ \text { TVDS } & \text { Transient voltage disturbance source } \\ \text { DP } & \text { Disturbance power } \\ \text { DE } & \text { Disturbance energy } \\ \text { DG } & \text { Distributed generator } \\ \text { IF } & \text { Information fusion } \\ \text { BPAF } & \text { Basic probability assignment function } \\ \text { GWN } & \text { Gaussian white noise } \\ \text { TM } & \text { Traditional matrix-based method } \\ \text { DM } & \text { Disturbance measuring method }\end{array}$

\section{References}

1. Milanovic, J.V.; Meyer, J.; Ball, R.F.; Howe, W.; Preece, R.; Bollen, M.H.J.; Elphick, S.; Cukalevski, N. International Industry Practice on Power-Quality Monitoring. IEEE Trans. Power Deliv. 2014, 29, 934-941.

2. Liu, Z.; Cui, Y.; Li, W. A Classification Method for Complex Power Quality Disturbances Using EEMD and Rank Wavelet SVM. IEEE Trans. Smart Grid 2015, 6, 1678-1685.

3. Huang, N.; Zhang, S.; Cai, G.; Xu, D. Power Quality Disturbances Recognition Based on a Multiresolution Generalized S-Transform and a PSO-Improved Decision Tree. Energies 2015, 8, 549-572.

4. Wang, M.H.; Yau, H.T. New Power Quality Analysis Method Based on Chaos Synchronization and Extension Neural Network. Energies 2014, 7, 6340-6357.

5. Chung, I.Y.; Won, D.J.; Kim, J.M.; Ahn, S.J.; Moon, S.I. Development of a network-based power quality diagnosis system. Electr. Power Syst. Res. 2007, 77, 1086-1094.

6. Tan, R.H.G.; Ramachandaramurthy, V.K. Voltage Sag Acceptability Assessment Using Multiple Magnitude-Duration Function. IEEE Trans. Power Deliv. 2012, 27, 1984-1990.

7. Demirci, T.; Kalaycioglu, A.; Kucuk, D.; Salor, O.; Guderl, M.; Pakhuylu, S.; Atalik, T.; Inan, T.; Cadirci, I.; Akkaya, Y.; et al. Nationwide real-time monitoring system for electrical quantities and power quality of the electricity transmission system. IET Gener. Transm. Distrib. 2011, 5, 540-550.

8. Won, D.J.; Chung, I.Y.; Kim, J.M.; Moon, S.I.; Seo, J.C.; Choe, J.W. A new algorithm to locate power-quality event source with improved realization of distributed monitoring scheme. IEEE Trans. Power Deliv. 2006, 21, 1641-1647.

9. Weng, G.; Zhang, Y.; He, H. A Novel Location Algorithm for Power Quality Disturbance Source Using Chain Table and Matrix Operation. Int. Rev. Electr. Eng. 2011, 6, 2746-2753.

10. Bentley, E.C.; Putrus, G.A.; McDonald, S.; Minns, P. Power quality disturbance source identification using self-organising maps. IET Gener. Transm. Distrib. 2010, 4, 1188-1196.

11. Abu-Elanien, A.E.B.; Salama, M.M.A. A Wavelet-ANN Technique for Locating Switched Capacitors in Distribution Systems. IEEE Trans. Power Deliv. 2009, 24, 400-409.

12. Hong, Y.Y.; Chen, B.Y. Locating Switched Capacitor Using Wavelet Transform and Hybrid Principal Component Analysis Network. IEEE Trans. Power Deliv. 2007, 22, 1145-1152.

13. Parsons, A.C.; Grady, W.M.; Powers, E.J.; Soward, J.C. A direction finder for power quality disturbances based upon disturbance power and energy. IEEE Trans. Power Deliv. 2000, 15, 1081-1086.

14. Zhang, W.; Wang, C. Transient disturbances location based on improved disturbance power and energy. Autom. Electr. Power Syst. 2007, 31, 32-35.

15. Kong, W.; Dong, X.; Chen, Z. Voltage sag source location based on instantaneous energy detection. Electr. Power Syst. Res. 2008, 78, 1889-1898.

16. Polajzer, B.; Stumberger, G.; Seme, S.; Dolinar, D. Detection of voltage sag sources based on instantaneous voltage and current vectors and orthogonal Clarke's transformation. IET Gener. Transm. Distrib. 2008, 2, 219-226.

17. Chen, P.C.; Malbasa, V.; Dong, Y.; Kezunovic, M. Sensitivity Analysis of Voltage Sag Based Fault Location With Distributed Generation. IEEE Trans. Smart Grid 2015, 6, 2098-2106. 
18. Alwash, S.F.; Ramachandaramurthy, V.K.; Mithulananthan, N. Fault-Location Scheme for Power Distribution System with Distributed Generation. IEEE Trans. Power Deliv. 2015, 30, 1187-1195.

19. Won, D.J.; Moon, S.I. Optimal Number and Locations of Power Quality Monitors Considering System Topology. IEEE Trans. Power Deliv. 2008, 23, 288-295.

20. Guo, Q.; Jia, Q.; Bo, Z. Locating power-quality event source through disturbance measures. In Proceedings of the 45th Universities Power Engineering Conference (UPEC), Cardiff, UK, 31 August-3 September 2010; pp. 1-5.

21. Huang, F.; Weng, G.; Wang, Q. Location of Power Quality Disturbance Source in Distribution Network with Distributed Generators. Autom. Electr. Power Syst. 2015, 39, 150-155.

22. Sun, K.; Chen, Q.; Gao, Z. An Automatic Faulted Line Section Location Method for Electric Power Distribution Systems Based on Multi-source Information. IEEE Trans. Power Deliv. 2015, 99, 1-10.

23. Farzanehrafat, A.; Watson, N.R. Power Quality State Estimator for Smart Distribution Grids. IEEE Trans. Power Syst. 2013, 28, 2183-2191.

24. Jin, T.; Chu, F.; Ling, C.; Nzongo, D.L.M. A Robust WLS Power System State Estimation Method Integrating a Wide-Area Measurement System and SCADA Technology. Energies 2015, 8, 2769-2787.

25. He, G.; Dong, S.; Qi, J.; Wang, Y. Robust State Estimator Based on Maximum Normal Measurement Rate. IEEE Trans. Power Syst. 2011, 26, 2058-2065.

26. Ray, P.K.; Kishor, N.; Mohanty, S.R. Islanding and Power Quality Disturbance Detection in Grid-Connected Hybrid Power System Using Wavelet and S-Transform. IEEE Trans. Smart Grid 2012, 3, 1082-1094.

27. Xia, M.; Li, X. Design and Implementation of a High Quality Power Supply Scheme for Distributed Generation in a Micro-Grid. Energies 2012, 6, 4924-4944.

28. Li, Z.; Yin, X.; Zhang, Z.; He, Z. Wide-Area Protection Fault Identification Algorithm Based on Multi-Information Fusion. IEEE Trans. Power Deliv. 2013, 28, 1348-1355.

29. Murphy, C.K. Combining belief functions when evidence conflicts. Decis. Support Syst. 2000, 29, 1-9.

30. IEEE PES Distribution System Analysis Subcommittee's Distribution Test Feeder Working Group. Distribution Test Feeders. Available online: http://ewh.ieee.org/soc/pes/dsacom/testfeeders/2010 (accessed on 17 September 2010).

(C) 2016 by the authors; licensee MDPI, Basel, Switzerland. This article is an open access article distributed under the terms and conditions of the Creative Commons Attribution (CC-BY) license (http://creativecommons.org/licenses/by/4.0/). 